\title{
Magnetic field influence on aurorae and the Jovian plasma disk radial structure
}

\author{
E. S. Belenkaya ${ }^{1}$, P. A. Bespalov ${ }^{2}$, S. S. Davydenko ${ }^{2}$, and V. V. Kalegaev ${ }^{1}$ \\ ${ }^{1}$ Institute of Nuclear Physics, Moscow State University, Moscow, Russia \\ ${ }^{2}$ Institute of Applied Physics RAS, Nizhny Novgorod, Russia
}

Received: 7 July 2005 - Revised: 7 February 2006 - Accepted: 13 March 2006 - Published: 19 May 2006

\begin{abstract}
The Jovian paraboloid magnetospheric model is applied for the investigation of the planet's auroral emission and plasma disk structure in the middle magnetosphere. Jupiter's auroral emission demonstrates the electrodynamic coupling between the ionosphere and magnetosphere. For comparison of different regions in the ionospheric level and in the magnetosphere, the paraboloid model of the global magnetospheric magnetic field is used. This model provides mapping along highly-conducting magnetic field lines. The paraboloid magnetic field model is also applied for consideration of the stability of the background plasma disk in the rotating Jupiter magnetosphere with respect to the flute perturbations. Model radial distribution of the magnetic field and experimental data on the plasma angular velocity in the middle Jovian magnetosphere are used. A dispersion relation of the plasma perturbations in the case of a perfectly conducting ionosphere is obtained. Analyzing starting conditions of a flute instability in the disk, the "threshold" radial profile of the plasma density is determined. An application of the results obtained to the known data on the Jovian plasma disk is discussed.
\end{abstract}

Keywords. Magnetospheric physics (Planetary magnetospheres; Polar cap phenomena; Solar wind-magnetosphere interactions)

\section{Introduction}

Contrary to the case of the Earth, where the energy of the auroral emission is ultimately taken from the energy of the solar wind, the energy of Jupiter's aurora is obtained mainly from the planetary rotation energy. For the outer planets, the rotation period varies as a function of latitude, so it has been necessary to define several longitude systems. System I corresponds to the rotation of equatorial latitudes $\left( \pm 10^{\circ}\right)$,

Correspondence to: E. S. Belenkaya

(elena@dec1.sinp.msu.ru) while System II relates to higher latitudes which rotate more slowly. System III has been defined to take into account the rotation period of the magnetic field. The Hubble Space Telescope (HST) images of Jupiter's aurora exhibit three distinct regions with independent variations in time: (1) the satellite footprint emissions, (2) the bright main oval emissions, and (3) all other emissions poleward of the main oval, the socalled polar emissions (Grodent et al., 2003a, b; Cowley et al., 2003). A long-term comparison of the images shows that the bulk of the auroral morphology is fixed in System III and reminded stable over a 5-year period (Grodent et al., 2003a).

A bright ring which corresponds to the main oval was described in Clarke et al. (1996, 1998), and in Prangé et al. (1998). Pallier and Prangé (2001) noted that in both Jovian hemispheres, the north and south main oval emissions appear conjugate, and defined the northern and southern reference main ovals (see, also Clarke et al., 1996, 1998, and Prangé et al., 1998). Pallier and Prangé (2001) concluded that the main oval is a very narrow structure which surrounds each pole of Jupiter approximately along the footprints of magnetic field lines crossing the equator in the middle magnetosphere near $\sim 20-30 R_{J}$ on the dayside, and maybe somewhat closer in on the nightside $\left(R_{\mathrm{J}} \approx 7 \cdot 10^{7} \mathrm{~m}\right.$ is Jupiter's radius).

Ballester et al. (1996) observed that the main oval emissions corotate with Jupiter. Clarke et al. (1998) noted that the main auroral oval not only rotates with the planet but also displays systematic addition motions. Grodent et al. (2003a) mentioned sub-corotation of the main oval (or of some individual auroral features of it). Bunce and Cowley (2001), Hill (2001), and Southwood and Kivelson (2001) suggested that the main oval is connected with the magnetosphere-ionosphere coupling current system associated with the breakdown of rigid corotation in the middle Jovian magnetosphere. Thus, the main auroral oval may be interpreted as the ionospheric footprint of the upward fieldaligned current caused by the breakdown of rigid corotation.

Published by Copernicus GmbH on behalf of the European Geosciences Union. 
Significant sub-corotation of the plasma flow was found on the ionospheric latitudes higher than the main oval (Rego et al., 1999) (the Earth's high-latitude auroral pattern is fixed with respect to the solar wind direction). Stallard et al. (2001) derived a detailed infrared picture of the auroral morphology in this region. They identified a dark (in the UV) polar region on the dawn side and a bright polar region on the dusk side. Stallard et al. (2001) presented Doppler observations within both of these polar regions and found that the emission in the bright polar region is weakly sub-corotational, while in the dark polar region there is a strong sub-corotation. Most recently, Stallard et al. (2003) found that the dark polar region may be divided into two separate regions: the fixed dark polar region which is near stagnant in the magnetic pole reference frame, and the rotating dark polar region (which subcorotates with the planet), located between the fixed dark polar region and the main oval. Based on those observations, Cowley et al. (2003) proposed a general structure of the equatorial and ionospheric plasma flows. Since the region of open field lines is magnetically connected to a tail lobe with very low plasma density, it is expected to be auroraly dark. According to the Cowley et al. (2003) picture, the fixed dark polar region is identified with the stagnant open field line region.

Grodent et al. (2003a) mentioned that the polar emissions are more variable than the main oval ones (their character time may reach tens of seconds, while the main oval variations are on time scales of tens of minutes to hours, and that the main oval usually contributes $\sim 70 \%$ of the total auroral emission). Prangé et al. (2001) noted that the intensities of the northern and southern aurora are very tightly correlated, although their brightnesses differ in absolute value. This tight and quantitative interhemispheric correlation suggest that the dominant auroral processes take place on closed field lines. Prangé et al. (1998) described fainter, roughly concentric, and very narrow ovals detected poleward of the north main oval. Their brightness is not related to the main oval activity. Prangé et al. (1998) suggested that the inner ovals are on open field lines of the tail, maybe near the polar cap boundary. Detailed investigation of the Jovian polar cap had been further developed in Pallier and Prangé (2001). They noted that the transpolar emission crosses the polar region from one side of the main oval to the other. Pallier and Prangé (2001) also suggested that the poleward edge of the transpolar emission and the innermost inner oval generally resemble a high-latitude limit for bright auroral emissions, thus representing west and east parts of the Jovian northern polar cap boundary. Pallier and Prangé (2001) noted that the polar cap shape and size vary as a function of the solar wind and interplanetary magnetic field (IMF) conditions.

Jupiter's magnetospheric magnetic field model is necessary to clarify the regions in the magnetosphere connected with specific features in the high-latitude Jovian ionosphere. The models most often used were developed by Connerney (1992) and Connerney et al. (1998) (see, e.g. Prangé et al.,
1998; Tomas et al., 2004), or by Khurana (1997) which supplements one of Connerney's (1993) internal field models with a warped and twisted current sheet (see, e.g. Prangé et al., 2001). However, it should be noted that Connerney's (1998) VIP4 model is not applicable beyond $30 R_{J}$. At the same time, just these outer parts of the Jovian magnetosphere are responsible for the polar aurora emissions.

Khurana's (1997) model couples the internal field spherical harmonic coefficients from the Goddard Space Flight Center $\mathrm{O}_{6}$ model with an Euler potential formulation of the external field. This model cannot be used in the outer dayside magnetosphere and at higher latitudes at any local time because the magnetopause currents are not included. The magnetopause currents were included in the Engle (1991, 1992a, b) model. The magnetic field of the magnetopause currents was added to those of a model current system in the equatorial plane and the intrinsic dipole field of Jupiter. (Engle and Beard (1980) assumed that the contribution to the total field due to the magnetopause currents is a negligible fraction of the total field near the equator at about midsheet.) However, the magnetotail contribution had not been included. Moreover, all of these models ignored the IMF effects. Walker and Ogino (2003) used a global magnetohydrodynamic simulation of the interaction of Jupiter's magnetosphere with the solar wind to study the solar wind effects on the structure of currents in the Jovian magnetosphere. They used an image dipole to help form a magnetospheric cavity. The Jovian magnetospheric currents were simulated for southward and northward IMF.

Fukazawa et al. (2005) also used a time dependent, 3-D, magnetohydrodynamic simulation of the interaction between the Jovian magnetosphere and the solar wind to investigate the importance of the IMF.

However, there is no global Jovian magnetospheric model (except MHD simulations) which takes into account all large-scale magnetospheric current systems (including magnetotail) as well as the interplanetary magnetic field penetrating into the magnetosphere. Presently, even locations of closed and open field line areas are under debate. For these reasons the paraboloid magnetospheric Jovian model described in Sect. 2 was constructed (Belenkaya, 2003, 2004; Alexeev and Belenkaya, 2005), on the basis of the terrestrial paraboloid magnetospheric model (Alexeev, 1986). This global large-scale magnetospheric magnetic field model allows us to establish the correlation of the most high-latitude ionospheric regions with different regions and processes in the distant magnetosphere. The paraboloid model also includes the interplanetary magnetic field effects.

The Jovian magnetosphere is very dynamic, although it is not clear whether this variability is due to an external influence of the solar wind or to internal effects related to plasma transport from Io (Prangé et al., 2001). The mutual relation between the solar wind and internal magnetospheric processes in their influence on the Jovian aurora is not well understood now. One of the aims of the present paper is an 
attempt to clarify some points of this relation and to use the constructed model for this goal (see Sect. 3).

The model of the global magnetospheric magnetic field is also very significant for the analysis of the plasma distribution and stability in the magnetosphere. As known, the Pioneer 10 investigation of the Jupiter's magnetosphere shows that background plasma and energetic particles are confined to a narrow latitudinal range in the vicinity of the magnetic equator with relatively small asymmetries in longitude (Smith et al., 1974; McKiben and Simpson, 1974). To explain the observed particle flux and plasma distribution, a model of the thin plasma disk in the middle Jovian magnetosphere has been suggested. Voyager 1 and 2 provided additional data on the magnetic field, as well as measurements of plasmas not obtainable by Pioneer 10. The Jovian plasma disk was observed by a number of experiments on board both Voyager spacecraft, including a plasma science experiment (Bridge et al., 1979a, b) and the low-energy charged particle experiment (Krimigis et al., 1979a).

As shown by Walker et al. (1978), the thermal pressure in the middle magnetosphere is primarily due to the suprathermal ion component with characteristic energies about $25 \mathrm{keV}$ and above, whereas the mass densities are dominated by ions with energies of less than $6 \mathrm{keV}$. Later, these conclusions were confirmed by direct measurements (Krimigis et al., 1979a, b). Thus, there are two interconnected plasma disks in the Jovian magnetosphere: the current disk of the suprathermal particles, providing mainly the energy density of the magnetodisk, and the plasma sheet of the low-energy ion component, dominating in the mass density. The weakening of the magnetic field in the vicinity of the current disk promotes the accumulation of cold and heavy ions there under the action of centrifugal force, and one can suppose that current and plasma disks mainly coincide.

Here, using the magnetic field model, we consider a radial density profile of the comparatively low-pressure background plasma in the Jovian magnetodisk. Since the kinetic pressure and current systems caused by the instabilities of the background plasma disturb only slightly the magnetospheric magnetic field, we can use the given field approximation. It is known that the pattern of the magnetic field exerts a significant influence on the structure of the background plasma disk: the region of the weak magnetic field in the vicinity of the magnetodisk mainly corresponds to the location of the relatively cold plasma disk, whereas the profile of the magnetic field strength in the disk plane affects the radial distribution of the plasma density.

In Sect. 4 we analyze in more detail the flute instability in the plasma disk as applied to the formation of the radial profile of the plasma density. As follows from a number of studies (see, e.g. Melrose, 1967; Bespalov and Davydenko, 1994; Liu, 1998), the most significant factors exerting an influence on the development of the flute instability are the structure of the magnetic field in the disk, and the high conductivity of the planetary ionosphere, which connects magnetic flux tubes. We assume the structure of the magnetic field in the disk according the suggested model, and study in more detail the influence of the conducting ionosphere on the radial distribution of the plasma density, taking into account the influence of the rarified plasma between the disk boundaries and the ionosphere.

\section{The Jovian paraboloid magnetospheric magnetic field model}

The main contributors to the magnetospheric magnetic field in the paraboloid model are (Alexeev and Belenkaya, 2005):

1. The intrinsic magnetic (dipole) field, as well as the shielding magnetopause currents, which confine the dipole field inside the magnetosphere of Jupiter.

2. The tail currents and their closure currents on the magnetopause.

3. The disk current and the corresponding shielding magnetopause current.

4. The IMF penetrated into the magnetosphere.

The following equations for the magnetic field and electric current density:

$\operatorname{div} \mathbf{B}=0, \quad \operatorname{div} \mathbf{j}=0$

are true for all model calculations. Each magnetospheric current system conserves the condition $B_{n}=0$ at the magnetopause. Both the magnetopause currents shielding all magnetospheric magnetic field sources, including distant tail, as well as the IMF penetrating into the magnetosphere, represent new elements in comparison with the other Jovian models. Flowing pass the obstacle (magnetosphere), the magnetic field of the solar wind drapes around it and, consequently, increases in the magnetopause vicinity. Increased diffusion, due to the field growth near the magnetospheric boundary, leads to the penetration of the IMF through the magnetopause inside the magnetosphere.

The magnetospheric magnetic field vector $\mathbf{B}$ was calculated in the Jovian solar-magnetospheric coordinate system:

$$
\begin{aligned}
& \mathbf{B}=\mathbf{B}_{d}(\psi)+\mathbf{B}_{T S}\left(\psi, R_{s s}, R_{2}, B_{\mathrm{t}}\right) \\
& +\mathbf{B}_{M D}\left(\psi, B_{D C}, R_{D 1}, R_{D 2}\right)+\mathbf{B}_{s d}\left(\psi, R_{s s}\right) \\
& +\mathbf{B}_{\mathrm{sMD}}\left(\psi, R_{s s}, B_{\mathrm{DC}}, R_{D 1}, R_{D 2}\right) \\
& +\mathbf{b}\left(\kappa_{\mathrm{J}}, \mathbf{B}_{I M F}\right) .
\end{aligned}
$$

Here $\mathbf{B}_{d}(\psi)$ is the dipole magnetic field; the field of the magnetospheric tail current system (cross-tail currents and their closure magnetopause currents) is $\mathbf{B}_{T S}\left(\psi, R_{s S}, R_{2}, B_{\mathrm{t}}\right)$; a field of the thin current disk placed near the equatorial plane is $\mathbf{B}_{M D}\left(\psi, B_{D C}, R_{D 1}, R_{D 2}\right)$; the field of currents on the magnetopause shielding the dipole field is $\mathbf{B}_{s d}\left(\psi, R_{s s}\right) ; \mathbf{B}_{\mathrm{sMD}}\left(\psi, R_{s s}, B_{\mathrm{DC}}, R_{D 1}, R_{D 2}\right)$ is the field of the currents on the magnetopause shielding the disk current 
field; $\mathbf{b}\left(\kappa_{\mathrm{J}}, \mathbf{B}_{I M F}\right)$ is a part of the interplanetary magnetic field penetrating into the magnetosphere. Here we consider the tail current sheet and the current disk to be infinitely thin. On the night-side the magnetodisk merges with the magnetotail current system.

For calculation of the Jovian magnetospheric magnetic field (Eq. 2), the input model parameters should be defined: the magnetic dipole tilt angle, $\psi$ (the angle between the $Z$ axis of the Jovian solar-magnetospheric coordinate system and the dipole axis, here we consider $\psi=0$ ); the distance from Jupiter's center to the subsolar point on the magnetopause, $R_{S s}$; the distances to the outer and inner edges of the magnetodisk, $R_{D 1}$ and $R_{D 2}$, respectively; the distance from the planet's center to the inner edge of the magnetospheric tail current sheet, $R_{2}$; the magnitude of the field of the tail currents at the inner edge of the tail current sheet, $B_{\mathrm{t}} / \alpha_{0}, \alpha_{0}=\sqrt{1+2 R_{2} / R_{\mathrm{Ss}}} ;$ a magnetic field strength caused by the magnetodisk currents at the outer edge of the current disk, $B_{\mathrm{DC}}$; the interplanetary magnetic field vector, $\mathbf{B}_{I M F}$, and the coefficient of its penetration into the magnetosphere, $\kappa \mathrm{J}$.

Caudal (1986) showed that a self-consistent model of Jupiter's disk, including the effects of centrifugal force and pressure, gives $1 / r$ magnetodisk current dependence. However, here, following Barish and Smith (1975) and Beard and Jackson (1976), we assume a $1 / r^{2}$ current disk dependence. In this case, the magnetic flux of the disk field, $F l_{\mathrm{MD}}=B_{\mathrm{MD}} r \cdot 2 \pi r^{2}$, across the Southern or Northern Hemisphere is constant. As it is shown by Alexeev and Belenkaya (2005), this dependence, for example, fits well to the Ulysses data.

Here we will demonstrate applications of the Jovian paraboloid magnetospheric model for investigations of the auroral phenomena and a radial plasma distribution in the magnetodisk. In prepartion, we review the mathematic description of the magnetodisk field used in the Jovian paraboloid magnetospheric model (Alexeev and Belenkaya, 2005). A spherical coordinate system is used with the axis $Z$ parallel to the dipole axis, the polar angle $\theta$, and the azimuthal angle $\varphi$, counted in the planet rotation direction. The rigid plasma disk is placed in the magnetic equatorial plane. The azimuthal symmetry is suggested about the magnetic dipole axis. The azimuthal magnetodisk current, $\mathbf{j}_{\mathrm{MD} \varphi}$, exists only inside the disk $\left(R_{D 1}>r>R_{D 2}\right)$ and is directed to dusk in the dayside, and to dawn in the nightside.

If we assume that in the magnetodisk only the azimuthal current, $\mathbf{j}_{\mathrm{MD} \varphi}$, exists, a vector-potential $\mathbf{A}_{\mathrm{MD}}$ of the magnetodisk magnetic field $\mathbf{B}_{\mathrm{MD}}\left(\mathbf{B}_{\mathrm{MD}}=\nabla \times \mathbf{A}_{\mathrm{MD}}\right)$ has only one nonzero component, $A_{\mathrm{MD}} \varphi$. In a current-free region, $A_{\mathrm{MD}} \varphi$ is a solution of equation: $\nabla \times \nabla \times A_{\mathrm{MD}} \varphi=0$, which in spherical coordinates looks like:

$r \frac{\partial^{2}\left(r A_{\mathrm{MD}}\right)}{\partial r^{2}}+\frac{\partial}{\partial \theta}\left(\frac{1}{\sin \theta} \frac{\partial\left(A_{\mathrm{MD} \varphi} \sin \theta\right)}{\partial \theta}\right)=0$.
Assuming a separation of variables, we can find solutions in the form

$$
\begin{aligned}
& r^{n} \cdot P_{n}^{1}(\cos \theta) \text { and } \frac{P_{n}^{1}(\cos \theta)}{r^{n+1}}, \\
& n=1,2, \ldots, \infty,
\end{aligned}
$$

where $P_{n}^{1}(\cos \theta)$ are the associated Legendre polynomial functions. These solutions provide a continuity of the magnetic field at the edges of the magnetodisk. Discontinuity of the magnetic field caused by the disk current is described by another solution of Eq. (3):

$A_{\mathrm{MD} \varphi}^{(1)}=B_{\mathrm{DC}} \frac{R_{\mathrm{D} 1}^{2}}{r}\left\{\begin{array}{l}\tan \frac{\theta}{2} \text { for } \quad 0 \leq \cos \theta, \\ \cot \frac{\theta}{2} \text { for } \quad \cos \theta \leq 0 .\end{array}\right.$

This solution yields a drop in $B_{\mathrm{MD}} r$ at the equatorial plane $(\theta=\pi / 2):\left.\left\{B_{\mathrm{MD}} r\right\}\right|_{\theta / 2}$.

To construct a solution for the magnetic field of the disk current we use a principle of superposition of solutions (expressions 4 and 5). The vector potential $A_{\mathrm{MD} \varphi}$ can be written as:

$$
A_{\mathrm{MD} \varphi}=\left\{\begin{array}{cc}
\sum_{k=0}^{\infty} F_{1 k}\left(\frac{R_{\mathrm{D} 1}}{r}\right)^{2 k+2} P_{2 k+1}^{1}(\cos \theta) & \text { for } R_{\mathrm{D} 1} \leq r, \\
A_{\mathrm{MD} \varphi}^{(1)}+\sum_{k=0}^{\infty} P_{2 k+1}^{1}(\cos \theta)\left[F_{2 k}\left(\frac{r}{R_{\mathrm{D} 1}}\right)^{2 k+1}\right. \\
\left.+G_{1 k}\left(\frac{R_{D 2}}{r}\right)^{2 k+2}\right] & \text { for } R_{\mathrm{D} 2}<r<R_{\mathrm{D} 1}, \\
\sum_{k=0}^{\infty} G_{2 k}\left(\frac{r}{R_{\mathrm{D} 2}}\right)^{2 k+1} & P_{2 k+1}^{1}(\cos \theta) \\
& \text { for } r \leq R_{\mathrm{D} 2} .
\end{array}\right.
$$

To calculate the coefficients $F_{1 k}, F_{2 k}$ and $G_{1 k}, G_{2 k}$, we use the continuity conditions for $B_{\mathrm{MD} \theta}$ and $B_{\mathrm{MD}} r\left(B_{\mathrm{MD} \varphi} \equiv 0\right.$ in the considered model) at the edges of the disk (at $r=R_{D 1}$ and $\left.r=R_{D 2}\right)$.

The resulting expression for $B_{\mathrm{MD} r}=\frac{\partial\left(A_{\mathrm{MD} \varphi} \sin \theta\right)}{r \sin \theta \partial \theta}$ is

$B_{\mathrm{MD} r}=$

$B_{\mathrm{DC}}\left\{\begin{array}{l}\sum_{k=0}^{\infty} a_{2 k}\left(1-\rho_{0}^{2 k+1}\right) \frac{P_{2 k+1}(\cos \theta)}{\rho^{2 k+3}} \\ \text { for } R_{\mathrm{D} 1 \leq r,} \\ \frac{\operatorname{sign}(\cos \theta)}{\rho^{2}}+\sum_{k=0}^{\infty} P_{2 k+1}(\cos \theta)\left(a_{2 k+2}\right. \\ \left.-a_{2 k} \frac{\rho_{0}^{2 k+1}}{\rho^{4 k+3}}\right) \rho^{2 k} \text { for } R_{\mathrm{D} 2 \leq r \leq R_{\mathrm{D} 1},} \\ \sum_{k=0}^{\infty} a_{2 k+2}\left(1-\frac{1}{\rho_{0}^{2 k+2}}\right) \rho^{2 k} P_{2 k+1}(\cos \theta) \\ \text { for } r \leq R_{\mathrm{D} 2} .\end{array}\right.$ 


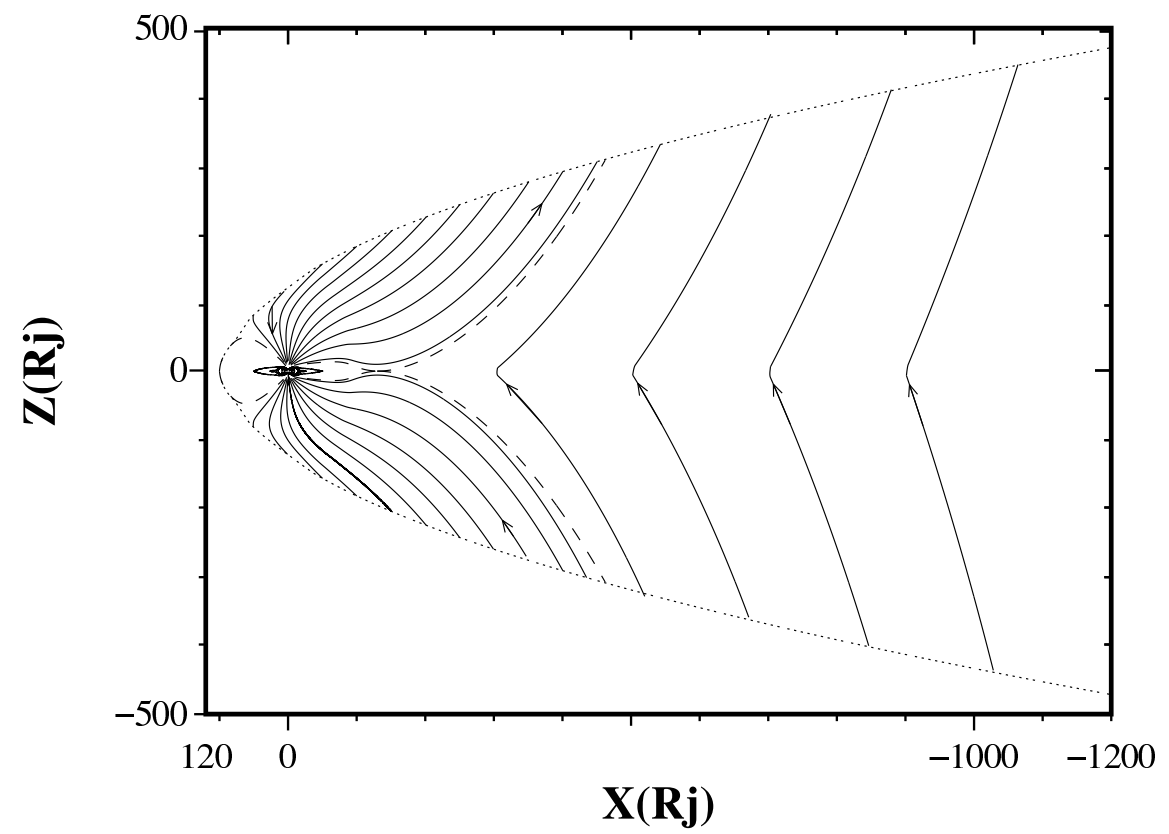

Fig. 1. Noon-midnight meridional cross section of the open Jovian magnetosphere for northward IMF $\left(B_{I M F x}=-0.02 \mathrm{nT} ; B_{I M F y}=0 \mathrm{nT}\right.$; $B_{I M F z}=0.62 \mathrm{nT}$ ) with $\kappa_{J}=0.8$. The model parameters are: $\psi=0 ; R_{S s}=100 R_{J} ; R_{2}=65 R_{J} ; B_{\mathrm{t}}=-2.5 \mathrm{nT} ; R_{D 1}=92 R_{J} ; R_{D 2}=18.4 R_{J}$; $B_{\mathrm{DC}}=2.5 \mathrm{nT}$. Dashed curves separate magnetic field lines of different topology types.

Here we introduce coefficients $a_{2 k}=\left.P_{2 k}(\cos \theta)\right|_{\theta=\pi / 2}=$ $\frac{(-1)^{k}}{2^{k} k !} 1 \cdot 3 \cdots(2 k-1)$, a dimensionless distance $\rho=\frac{r}{R_{\mathrm{D} 1}}$, and $\rho_{0}=\frac{R_{\mathrm{D} 2}}{R_{\mathrm{D} 1}}$. Function sign $(\cos \theta)$ could be expanded in a series of Legendre polynomials:

$\operatorname{sign}(\cos \theta)=\sum_{k=0}^{\infty} \frac{a_{2 k}}{2 k+2} P_{2 k+1}(\cos \theta)$,

where calculations were performed for $k \leq 50$. $B_{\mathrm{MD} \theta}=-\frac{\partial\left(r A_{\mathrm{MD} \varphi}\right)}{r \partial r}$ can be expressed as

$B_{\mathrm{MD} \theta}=$

$B_{\mathrm{DC}}\left\{\begin{array}{c}\sum_{k=0}^{\infty} \frac{a_{2 k}}{2 k+2}\left(1-\rho_{0}^{2 k+1}\right) \frac{P_{2 k+1}^{1}(\cos \theta)}{\rho^{2 k+3}} \\ \text { for } R_{\mathrm{D} 1} \leq r, \\ \sum_{k=0}^{\infty} \frac{a_{2 k}}{2 k+2}\left(\rho^{2 k}-\frac{\rho_{0}^{2 k+1}}{\rho^{2 k+3}}\right) P_{2 k+1}^{1}(\cos \theta) \\ \text { for } R_{\mathrm{D} 2} \leq r \leq R_{\mathrm{D} 1}, \\ \sum_{k=0}^{\infty} \frac{a_{2 k}}{2 k+2}\left(\begin{array}{c}\left.\rho_{0}^{2 k}-\frac{1}{\rho_{0}^{2}}\right) \frac{\rho^{2 k}}{\rho_{0}^{2 k}} P_{2 k+1}^{1}(\cos \theta) \\ \text { for } r \leq R_{\mathrm{D} 2} .\end{array}\right.\end{array}\right.$
The other component of the magnetodisk magnetic field

For $R_{D 1}<r$ the first term in the sum for $B_{\mathrm{MD} \theta}$ (Eq. 9) corresponding to $k=0$ and $\theta=\pi / 2$ is equal to

$$
\left.B_{\mathrm{MD} \theta}\right|_{k=0, \theta=\pi / 2}=-\frac{B_{\mathrm{DC}}}{2 \rho^{3}}\left(1-\rho_{0}\right)=\frac{M_{\mathrm{MD}}}{r^{3}},
$$

where

$M_{\mathrm{MD}}=\frac{B_{\mathrm{DC}}}{2} R_{\mathrm{D} 1}^{3}\left(1-\rho_{0}\right)$

is an effective magnetic moment of the magnetodisk field for $R_{D 1}<r$. As it was described by Belenkaya (2004), we chose the value of $R_{\mathrm{D} 2}$ as the distance where the field distortions associated with the current sheet become dominant. Following Barbosa et al. (1979) (who stated the existence of an extensive magnetoplasmadisk from 20 to $\sim 80-90 R_{\mathrm{J}}$ ) and Engle and Beard (1980) (using an equatorial current sheet from $\sim 18$ to $100 R_{\mathrm{J}}$ ), we took the equatorial current sheet from $\sim 18 R_{\mathrm{J}}$ to $R_{\mathrm{D} 1}=92 R_{\mathrm{J}}$. (These parameter values allow us to fit well to the Ulysses data from Alexeev and Belenkaya, 2005.) Below we consider two applications of the paraboloid model for calculations of the IMF effects on Jupiter's aurorae, and for study of plasma distribution in the Jovian magnetodisk. 


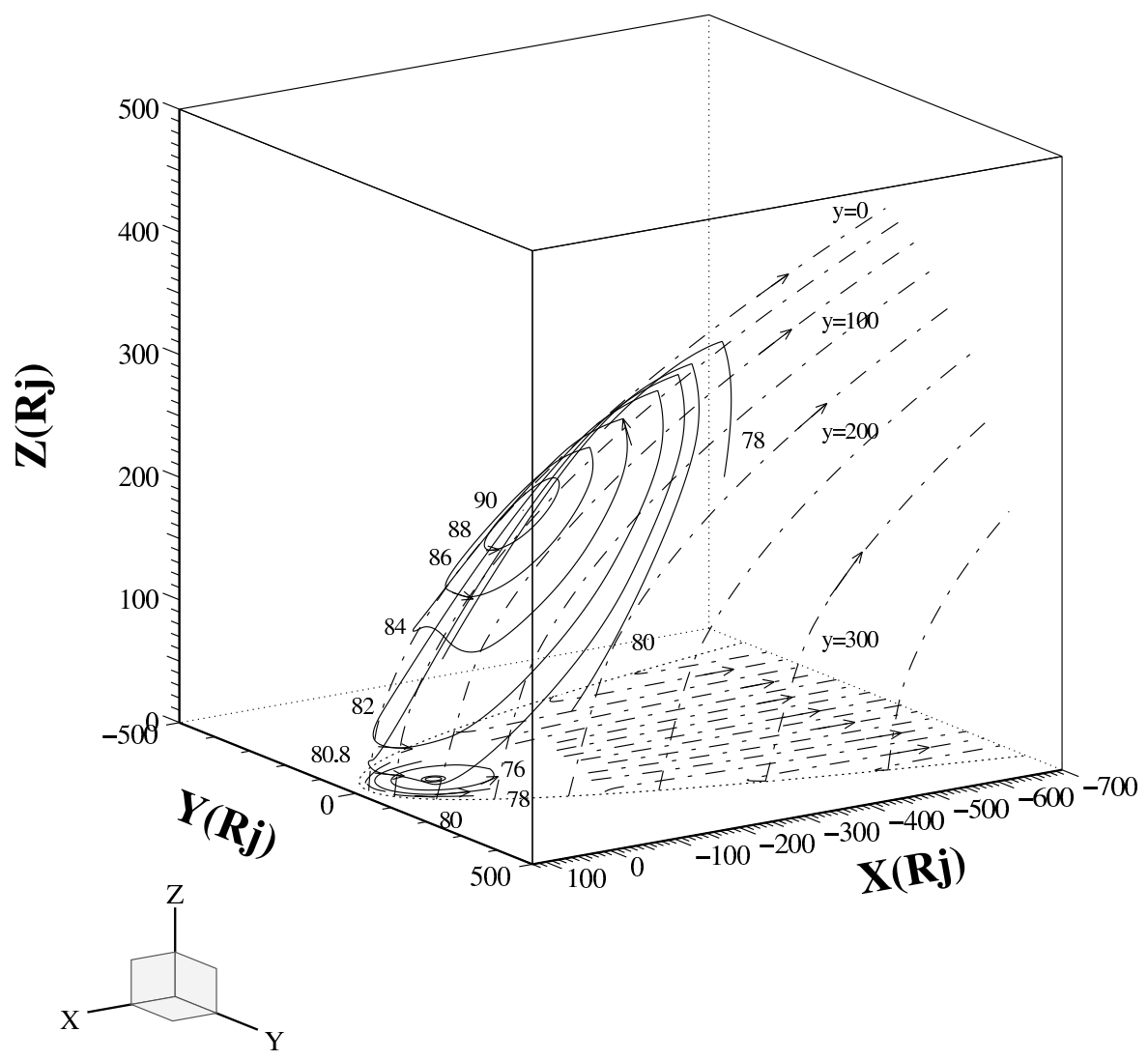

Fig. 2. Three-dimensional Jovian magnetosphere for northward IMF. Electric equipotentials of the corotation (solid curves) and solar wind (dashed-dotted curves) electric fields on the magnetopause and on the equatorial plane are shown. For the dashed-dotted curves, $\Delta y=50 R_{\mathrm{J}}$ and $\Delta U=0.75 \mathrm{MV}$. For the solid curves on the equatorial plane (from the outer to inner) the latitudes and electric corotation potentials are: $80^{\circ}, 0.4 \mathrm{MV} ; 78^{\circ}, 1.4 \mathrm{MV} ; 76^{\circ}, 4.3 \mathrm{MV} ; 74^{\circ}, 7.6 \mathrm{MV} ; 0^{\circ}, 356 \mathrm{MV}$, respectively. For the solid curves on the magnetopause (from the outer to inner) the corresponding values are: $81^{\circ}, 0 \mathrm{MV} ; 82^{\circ},-1.9 \mathrm{MV} ; 84^{\circ},-5.1 \mathrm{MV} ; 86^{\circ},-7.4 \mathrm{MV} ; 88^{\circ},-8.8 \mathrm{MV} ; 90^{\circ},-9.2 \mathrm{MV}$, respectively. IMF and model parameters are as for Fig. 1.

response of the Jovian magnetopause and bow shock positions to changes in the north-south IMF component. In spite of the fact that the magnetopause position is determined by the requirement of the pressure balance, a secondary control of this position is provided by the sense of the IMF. Kivelson and Southwood (2003) proposed that for Jupiter this process is analogous to that found at Earth but the effects of northward and southward IMF are reversed, as the Jovian dipole moment is antiparallel to that of Earth.

In spite of the large Jovian dipole moment and strong magnetodisk field, a penetrating solar wind magnetic field of very small strength occurs and is significant for the global magnetospheric topology. For northward IMF, the Jovian magnetospheric structure is simpler (as for the southward IMF for the Earth), and open field lines do not intersect the equatorial magnetospheric plane on their way to the polar caps (see Fig. 1). That is why the equatorial corotation lagging (due to the centrifugally driven radial outflow of the iogenic plasma concentrated in the equatorial plane, and conservation of angular momentum) is transferred only to the iono- spheric closed field line region, but not to the high-latitude ionospheric open field line region. Thus, for northward IMF, the electric field generated by Jupiter's rotation is transmitted along open field lines outward to enforce the corotation of magnetospheric plasma under the assumption that magnetic field lines are perfect conductors. Rigid corotation can exist up to the distance at which the azimuthal speed equals the Alfvén speed (beyond this distance the information about rigid corotation cannot be transmitted along field lines with Alfvén velocity). In the Jovian tail lobe the Alfvén velocity is much higher $\left(\sim 31 \cdot 10^{3} \mathrm{~km} \mathrm{~s}^{-1}\right.$ (Goldstein et al., 1985, 1986)) than in the equatorial plane $\left(\sim 200 \mathrm{~km} \mathrm{~s}^{-1}\right)$. Estimations done by Belenkaya (2004) showed that for northward IMF rigid corotation can be transmitted along open field lines up to the magnetopause (see Fig. 2).

Thus, for northward solar wind magnetic field, corotation may exist in the high-latitude ionosphere of both polar caps. This is why in this case, corotation determines the electric field distribution in most of the open field line regions in the Jovian ionosphere. Calculations performed in 


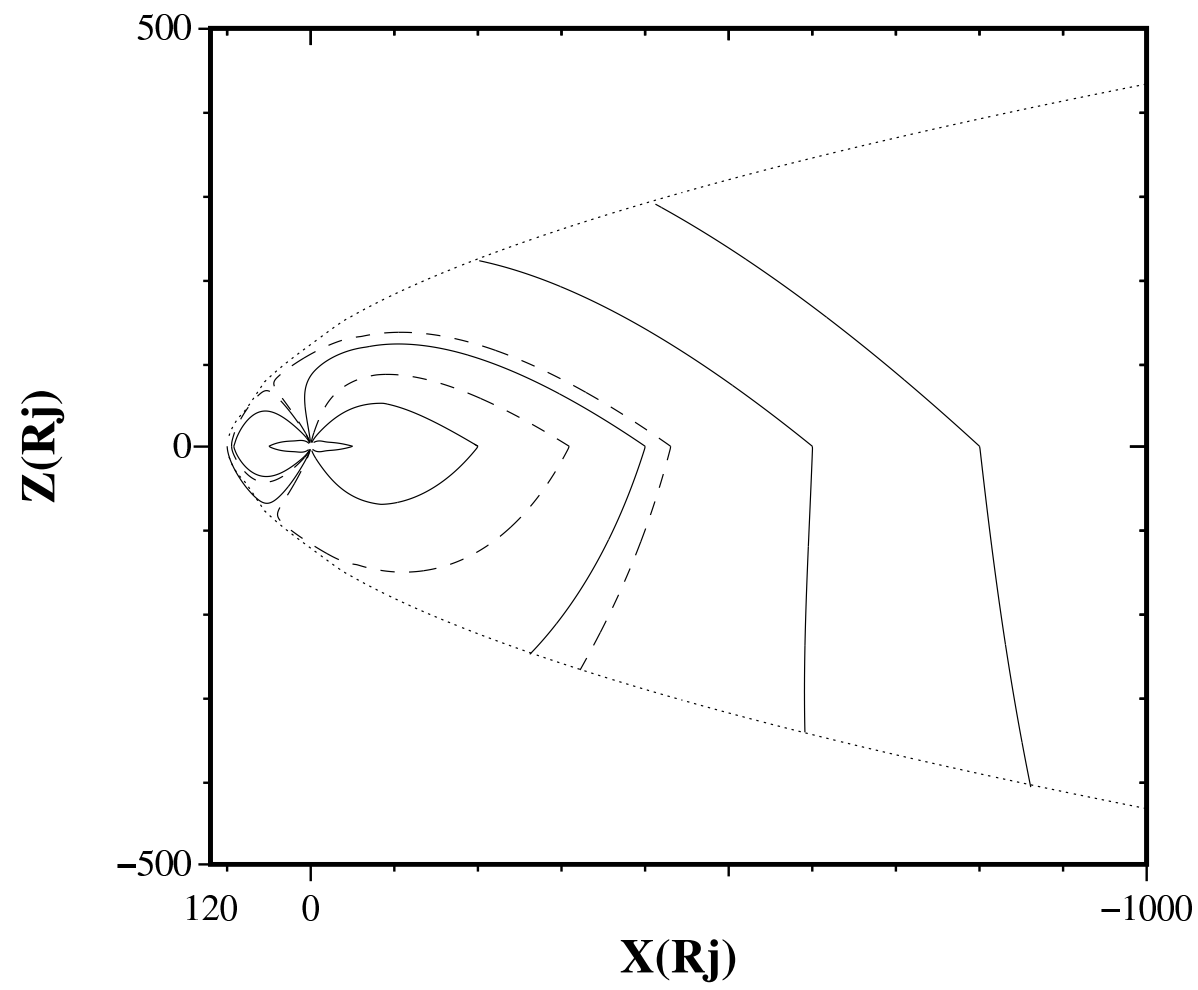

Fig. 3. Noon-midnight meridional cross section of the open Jovian magnetosphere for southward IMF $\left(B_{I M F x}=-0.26 \mathrm{nT} ; B_{I M F y}=0 \mathrm{nT}\right.$; $\left.B_{I M F z}=-0.5 \mathrm{nT}\right)$ with $k_{J}=0.8$. The model parameters are: $\psi=0 ; R_{S s}=100 R_{J} ; R_{2}=65 R_{J} ; B_{\mathrm{t}}=-2.5 \mathrm{nT} ; R_{D 1}=92 R_{J} ; R_{D 2}=18.4 R_{J}$; $B_{\mathrm{DC}}=2.5 \mathrm{nT}$. Dashed curves separate magnetic field lines of different topology types (closed, open, and the interplanetary magnetic field lines penetrating into the magnetosphere).

the Jovian paraboloid model for northward IMF with a penetrated $z$ component $0.5 \mathrm{nT}$ (Belenkaya, 2004) showed that only in a small spot near the pole (from $\sim 87^{\circ}$ to $90^{\circ}$ ) does the antisunward convection generated by the solar wind electric field prevail. According to these calculations, the ionospheric open field line region in the northern (southern) Jovian hemisphere is extended from $82.17^{\circ}\left(-82.33^{\circ}\right)$ at noon to $78.05^{\circ}\left(-77.59^{\circ}\right)$ at the midnight. Thus, the angular radius of the ionospheric open field line region is $\sim 10^{\circ}$. (Cowley et al. (2003) concluded that if the region of open flux was circular and centered on the magnetic pole, then it would occupy a region down to $\sim 9^{\circ}$ co-latitude.)

For the southward solar wind magnetic field, contrary to the case of northward IMF, open field lines go to the ionosphere after intersection with the magnetospheric equatorial plane (see Fig. 3). Using the Jovian paraboloid model and taking into account the observed lagging of corotation in the equatorial plane (caused by the radial plasma outflow and conservation of angular momentum), the corresponding potential of the corotation electric field was calculated in the Jovian ionosphere. The corotation braking in the equatorial magnetosphere leads to the partial stopping of a connected part of the ionosphere from corotating. According to $\mathrm{Be}-$ lenkaya $(2003,2004)$, the location of the ionospheric final corotation boundary is almost insensitive to the magnitude of the penetrating southward IMF field strength (there is no corotation on the ionospheric latitudes higher $\sim \pm 84^{\circ}$ ). It was also shown that for $\kappa_{J}$ varying from 0.1 to 1 and for reasonable values of southward IMF, the open field line region in the ionosphere is located in a zone free from corotation where reconnection effects should dominate (Belenkaya, 2003, 2004). For southward IMF, convection cells with sunward motion near the cusp projection arise on the highlatitude Jovian ionospheric open field lines (see Fig. 4). Here and in the subsequent text we neglect the $9.6^{\circ}$ offset between Jupiter's magnetic dipole axis and the spin axis.

Southwood and Kivelson (2001) and Cowley and Bunce (2001) argue that the auroral oval which maps to the part of the rotating flows falls below the corotation velocity. The main auroral ovals suggested to be connected with the regions of significant upward field-aligned currents (Hill, 2001) are associated with the field-aligned potential drops. In our model, the maximum field-aligned electric potential drop occurs at the latitudes corresponding to the locations of maximum magnetic field lines slippage: $L=R_{\mathrm{D} 2}$ (the corresponding ionospheric latitudes are $\sim \pm 74^{\circ}$, independent of the IMF orientation). This modeled position of the equatorward edge of the main oval is in good accordance with the 


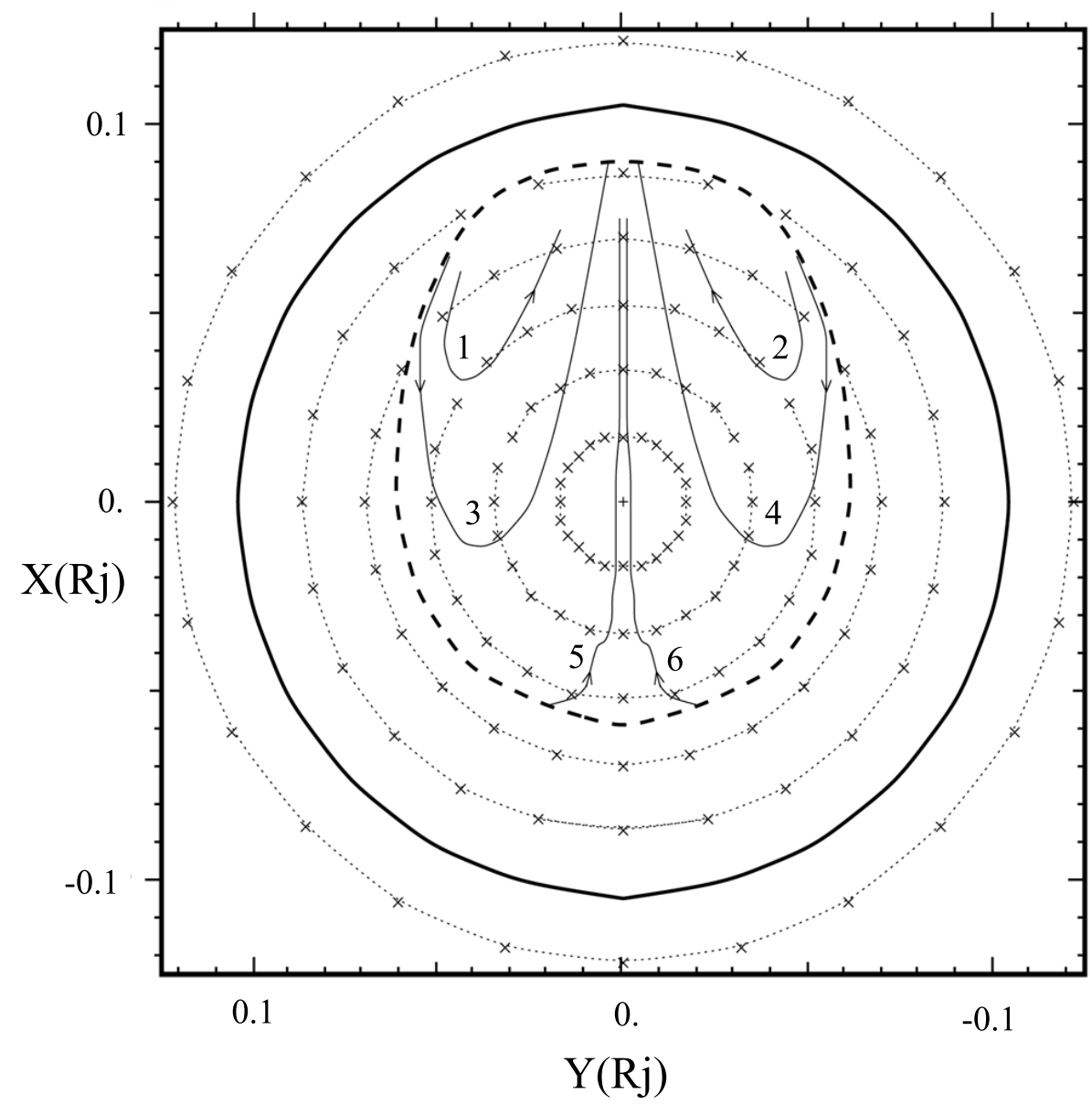

Fig. 4. The northern Jovian polar cap for southward IMF $\left(B_{I M F x}=-0.26 \mathrm{nT} ; B_{I M F y}=0 \mathrm{nT} ; B_{I M F z}=-0.5 \mathrm{nT}\right)$ with $\kappa_{J}=0.8$. The model parameters are: $\psi=0 ; R_{S S}=100 R_{J} ; R_{2}=65 R_{J} ; B_{\mathrm{t}}=-2.5 \mathrm{nT} ; R_{D 1}=92 R_{J} ; R_{D 2}=18.4 R_{J} ; B_{\mathrm{DC}}=2.5 \mathrm{nT}$. The Sun is on the top; positive $Y$ corresponds to the dusk side. Dotted lines with crosses mark lines of constant latitudes (from $\sim \pm 83^{\circ}$ to $\sim \pm 90^{\circ}$ ). The dashed curve marks the open field line boundary. The bold solid circle is the corotation boundary. The solid curves are equipotentials of the solar wind electric field: " 1 " corresponds to the potential $0.6 \mathrm{MV}$; "2" corresponds to $-0.6 \mathrm{MV}$; "3" corresponds to $0.3 \mathrm{MV}$; "4" corresponds to $-0.3 \mathrm{MV}$; "5" corresponds to $0.03 \mathrm{MV}$; and " 6 " corresponds to $-0.03 \mathrm{MV}$.

Connerney et al. (1998) and Cowley and Bunce (2001) results: the oval's angular radius is $\sim 16^{\circ}$.

In the inner part of the Jovian magnetosphere, the magnetic field guides space plasmas. Beyond the distance where the rigid corotation is finished, the plasma motion affects the strength and the configuration of the magnetic field. The length $L_{\mathrm{A}}$ represents the distance scale in the equatorial plane over which the rigid corotation breaks down. Beyond $L_{\mathrm{A}}$, the field should assume a disk-like geometry, and the plasma should lag behind the inner magnetosphere in its corotation.

In our model, $L_{\mathrm{A}}$ approximately coincides with the inner edge of the magnetodisk, $L_{\mathrm{A}} \sim R_{\mathrm{D} 2}$. According to observations (Kane et al., 1995, 1999), in the magnetodisk (for $\left.R_{\mathrm{D} 2} \leq L \leq R_{\mathrm{D} 1}\right)$ the angular velocity remains at roughly half the rigid corotation rate. We assume that beyond the magnetodisk, for $R_{\mathrm{D} 1} \leq L \leq R_{\mathrm{c}}$, the corotation significantly breaks down due to the action of the external forces or to the inertia of the corotating plasma; $R_{\mathrm{c}}$ is the equatorial boundary of the corotation region, out of which the corotation vanishes. At noon $R_{\mathrm{c}}$ may coincide with the distance to the subolar point or with the equatorial distance to the last closed field line. At the other LT, $R_{\mathrm{c}}$ is roughly approximated by the equatorial projection of the constant ionospheric latitude coincided with the footpoint of the noon field line with $L=R_{\mathrm{c}}$ (for southward IMF this ionospheric latitude is equal to $\pm 84^{\circ}$, and for northward IMF is equal to $\pm 81^{\circ}$ ). According to observations of the angular velocity in the outer dayside equatorial magnetosphere (for $R_{\mathrm{D} 1} \leq L \leq R_{\mathrm{c}}$ in our model), the speed of the azimuthal plasma flow is approximately one-fifth of the local rigid corotation speed (e.g. Laxton et al., 1997; Hawkins et al., 1998). So, for the crude estimation we suggest that in the equatorial section of the Jovian magnetosphere, the plasma corotates with an effective angular velocity $\Omega=\gamma \Omega_{\mathrm{J}}$, 
where $\gamma=1$ for $L \leq R_{\mathrm{D} 2}, \gamma=0.5$ for $R_{\mathrm{D} 2} \leq L \leq R_{\mathrm{D} 1}, \gamma=0.2$ for $R_{\mathrm{D} 1} \leq L \leq R_{\mathrm{c}}$, and $\gamma=0$ for $L \geq R_{\mathrm{c}}$. We suppose that due to the braking of the plasma rotation speed observed in the equatorial magnetosphere, velocities at the "ends" of the corresponding magnetic field lines (in the ionosphere) fall below the rigid corotation (in particular, the region of the equatorial plane where corotation is absent maps to the zone of the ionosphere prevented from rotating by the magnetosphere).

As it was mentioned above, the ionospheric projection of the final corotation boundary corresponds to the latitude $\sim \pm 84^{\circ}$ for southward IMF and to the latitude $\sim \pm 81^{\circ}$ for northward. So roughly, for southward IMF at the ionospheric latitudes less than $\sim \pm 74^{\circ}$ the rigid corotation exists; from $\sim \pm 74^{\circ}$ to $\sim \pm 84^{\circ}$ the sub-corotation should take place; at the latitudes higher than $\sim \pm 84^{\circ}$ the ionosphere does not rotate. For northward IMF, at the ionospheric latitudes less than $\sim \pm 74^{\circ}$ the rigid corotation exists; from $\sim \pm 74^{\circ}$ to $\sim \pm 81^{\circ}$ the sub-corotation should take place; for higher latitudes the rotation should be restored.

For northward IMF, according to our scenario, there is a corotation inside the Jovian ionospheric open field line region. For southward IMF ionospheric convection inside the open field line region is driven by the solar wind, and forms two cells for the IMF component $B_{I M F y}=0$ (see Fig. 4) and one vortex for $B_{I M F y} \neq 0$ (really for $B_{I M F y} \neq 0$ and $B_{I M F x} \neq 0$ there are two unequal cells, but one of them significantly prevails; for $B_{I M F X}=0$ there is only one vortex). Thus, dependent on the sign of $B_{I M F y}$ we can see, for example, on the dawn side of the northern ionospheric open field line region, the predominance of clockwise or anticlock-wise motions.

Cowley et al. (2003) suggested that the region of open field lines should be a region of near-stagnation in Jupiter's ionosphere in the rest frame of the dipole (that is in a frame where the dipole axis is at rest), compared with surrounding regions of sub-corotational flow. Cowley et al. (2003) also noted that the location and the stagnant nature of the fixed dark polar region may be associated with the region of open magnetic flux mapping to the tail lobes. In turn, Grodent et al. (2003b) stated that the location and shape of the so-called UV swirl region matches well the infrared fixed dark polar region observed by Stallard et al. (2003). The swirl region may be described as a region of faint, patchy, and short-lived (tens of seconds) emission features characterized by turbulent motions which occasionally form localized clockwise swirls, though the sense of rotation is not clear and varies from one data set to the next (Grodent et al., 2003b). It is located around the center of the polar region and fills approximately one-third of the area limited by the main oval. According to Grodent et al. (2003b), it is difficult to follow the motion of the faint, patchy, short-lived features which fill the swirl region, therefore one cannot determine to what extent it is corotating or not with the bulk of the auroral emission.

Following these suggestions, if we propose that the swirl region is associated with an open Jovian field line region, then the ratio $\eta$ of the area limited by the ionospheric projection of the inner edge of the magnetodisk to the area occupied by the ionospheric open field line region can be estimated and compared with the observed ratio of the area limited by the main oval to the swirl region area. Belenkaya (2003, 2004), using the paraboloid model, showed how the IMF controls Jupiter's magnetospheric structure and distribution at the ionospheric level of the open and closed field line regions. For the typical IMF strength at Jupiter's orbit, of the order of $0.5 \mathrm{nT}$, it was shown above that the radius of a circle approximating an open-closed field line boundary for northward IMF is $\sim 10^{\circ}$ (which coincides with the Walker and Ogino (2003) simulations result), and that a co-latitude of the ionospheric projection of the inner edge of the magnetodisk (imitating the equatorward boundary of the main oval in our model) is $\sim 16^{\circ}$. So, $\eta$ is of the order of 2.5 , which matches well the observed estimation of the swirl region area as one-third of the area limited by the main oval (for southward IMF of the same strength, the open field line region area on the Jovian ionosphere should be less). Thus, our model calculations of the ionospheric open field line region, controlled by the IMF, give results which in some sense are correspondent to the described observations of the swirl region: we obtained comparable areas of these objects, with variable rotating motions inside them.

The most bright auroral emissions in the Jovian ionosphere occur in the main oval. According, for example, to the Tomas et al. (2004) definition, the magnetospheric projection of the main Jovian oval is the equatorial region from $\sim 15 R_{J}$ to several tens of $R_{J}$, i.e. most of the magnetodisk. This is due to the fact that magnetic field above and below the disk is almost parallel to the equatorial plane, therefore a large region of the disk projects to a narrow ionospheric belt. Thus, the structure of the disk is directly connected with the processes responsible for the main oval emission. Below we will study a radial structure of the plasma density in the vicinity of the magnetodisk.

\section{The radial profile of the background plasma density in the magnetodisk}

Let us consider a radial distribution of background plasma density in the Jovian plasma disk in more detail. We restrict our consideration to the region $15 R_{\mathrm{J}} \leq r \leq 50 R_{\mathrm{J}}$, where $r$ is a distance from the planetary center. In this region, the centrifugal force in the plasma disk is mainly normal to the magnetic field lines and substantially exceeds the force of gravity. This makes prerequisites for the development of a flute instability, which has the greatest growth rate $\gamma$ and therefore is able to provide a most rapid transport of the cold iogenic plasma at the periphery of the magnetosphere. This flute instability as applied to the Jovian magnetospheric plasma transport was investigated in a number of articles. The first results (see, for example, Melrose, 1967) 
were mainly based on the a priori structure of the magnetospheric magnetic field and plasma properties and presented the linear approach to the problem. The following models used the results of the direct measurements of the magnetic field and charged particles in the Jovian magnetosphere (see Hill, 1976; Goertz, 1980, and others) and focused on the different aspects of the MHD stability of the plasma disk: an influence of the magnetic field perturbations on the development of the flute instability was treated by Liu (1998), a mixed magnetohydrodynamic-kinetic approach of low-frequency instabilities was suggested by Ferriere and Andre (2003), some aspects of the nonlinear diffusion of the iogenic plasma were considered, in particular, by Siscoe and Summers (1981). In most of the above considerations it was presumed that outside the disk proper a space is devoid of plasma, and communication between the boundary of the disk and the ionosphere is nearly instantaneous. Here we investigate the flute instability of the background magnetospheric plasma, taking into account both the effects of the well-conducting ionosphere and the regions of the rarified plasma outside the disk.

Since the plasma source in the inner magnetosphere is comparatively weak, we can follow the approach by Bespalov and Zheleznyakov (1990) and determine the equilibrium radial distribution of the plasma density in the disk, assuming it is at the threshold of the flute instability. Namely, we assume that at every radial distance the growth rate of the most unstable mode is equal to zero:

$\gamma_{\max }=0$

This estimation gives the steepest radial density profile, since an appearance of the unstable modes leads to the flute instability development which provides a fast transfer of the excess plasma to the periphery of the magnetosphere. This threshold profile can be further smoothed by more slow instabilities, but we do not consider these problems in this paper.

Since a flute instability arises due to a small difference between the drift velocities of ions and electrons, any factors which can influence this difference are of great interest. As shown in a large number of articles, one of the most significant factors is an inhomogeneity of the perturbations along the magnetic field. Here we consider an influence of this factor in more detail as applied to the background plasma disk in the Jovian magnetosphere.

\subsection{General dispersion relation in a plane layer}

To describe plasma processes we assume that any plasma component obeys the following set of two-fluid magnetohydrodynamic equations (the terms with kinetic pressure are omitted):

$\partial \bar{n}_{\alpha} / \partial t+\operatorname{div}\left(\bar{n}_{\alpha} \overline{\mathbf{v}}_{\alpha}\right)=0$,

$\bar{\rho}_{\alpha}\left(\partial \overline{\mathbf{v}}_{\alpha} / \partial t+\left(\overline{\mathbf{v}}_{\alpha} \nabla\right) \overline{\mathbf{v}}_{\alpha}\right)=q_{\alpha} \bar{n}_{\alpha}\left(\overline{\mathbf{E}}_{+} \overline{\mathbf{v}}_{\alpha} \times \mathbf{B} / c\right)+\bar{\rho}_{\alpha} \mathbf{g}_{\alpha}$, $\operatorname{div} \overline{\mathbf{E}}=4 \pi e\left(\bar{n}_{\mathrm{i}}-\bar{n}_{\mathrm{e}}\right)$

$\operatorname{rot} \overline{\mathbf{E}}=0$

Here $\alpha=\mathrm{i}$ corresponds to ions, and $\alpha=\mathrm{e}$ corresponds to electrons; $\bar{\rho}_{\alpha}, \bar{n}_{\alpha}$, and $\overline{\mathbf{v}}_{\alpha}$ are total density, concentration, and velocity of the corresponding plasma component, respectively, $q_{\mathrm{e}}=-e, q_{\mathrm{i}}=e, \overline{\mathbf{E}}$ and $\mathbf{B}$ are the electric and magnetic fields, respectively, $\bar{\rho}_{\alpha} \mathbf{g}_{\alpha}$ is a total force acting upon the unit volume of the corresponding plasma component, $c$ is the velocity of light.

Let us enter the local Cartesian coordinates in the disk (see Fig. 5), assuming, for simplicity, that the magnetic field lines are straight and directed along the $Z$ axis (the influence of their curvature is discussed below). A steady-state flow can be determined assuming $\partial / \partial t=0$ in the equations (Eqs. 13a and 13b), where $n_{\alpha}^{(0)}=n_{\alpha}^{(0)}(x), \mathbf{B}=B(x) \mathbf{z}_{0}$, and $\mathbf{g}_{\alpha}=g_{\alpha}(x) \mathbf{x}_{0}$. We also assume that the flow is uniform along the $Y$ axis and along the magnetic field, i.e. $\partial / \partial y=0, \partial / \partial z=0$. Since the rotation of real plasma disk is not rigid, it seems convenient to use a local co-rotating frame of reference, where using the quasineutrality of the medium $n_{\mathrm{i}}^{(0)}=n_{\mathrm{e}}^{(0)}$ one can obtain $\mathbf{E}^{(0)} \equiv 0$. So, at the given distance the rotation of the disk as a whole is absent, and the corresponding centrifugal force appears. Assuming that the medium does not move along the $X$ axis and along the magnetic field $\left(v_{\alpha x}^{(0)}=0\right.$ and $\left.v_{\alpha z}^{(0)}=0\right)$, Eq. (13b) gives a steady-state flow:

$\mathbf{v}_{\alpha}^{(0)}=\left\{0,-g_{x \alpha} \frac{m_{\alpha} c}{q_{\alpha} B}, 0\right\}$.

Let us consider perturbations in the form of $\exp \left[i\left(\omega t-k_{y} y-k_{z} z\right)\right]$, which can provide the greatest increments of flute instability (Mikhailovskii, 1974). Perturbed velocity and concentration of the $\alpha$-component are $\overline{\mathbf{v}}_{\alpha}=\mathbf{v}_{\alpha}^{(0)}+\mathbf{v}_{\alpha}$ and $\bar{n}_{\alpha}=n_{\alpha}^{(0)}+n_{\alpha}$, respectively; the perturbed electric field is $\mathbf{E}=-\nabla \psi=i \mathbf{k} \psi$. The continuity equation (13a) gives

$n_{\alpha}=\frac{i \operatorname{div}\left(n^{(0)} \mathbf{v}_{\alpha}\right)}{\omega-k_{y} v_{\alpha y}^{(0)}}$.

Equation (13b) gives

$i \omega \mathbf{v}_{\alpha}+\left(\mathbf{v}_{\alpha} \nabla\right) \mathbf{v}_{\alpha}^{(0)}+\left(\mathbf{v}_{\alpha}^{(0)} \nabla\right) \mathbf{v}_{\alpha}=\frac{q_{\alpha}}{m_{\alpha}}\left(i \mathbf{k} \psi+\frac{1}{c} \mathbf{v}_{\alpha} \times \mathbf{B}\right)+\mathbf{g}_{\alpha}$.

As seen, Eq. (16) can be divided into the separate equations for the field-aligned (longitudinal) and transverse to the magnetic field components of the perturbed velocity $v_{\alpha z}$ and $\mathbf{v}_{\alpha \perp}$, respectively. Namely, multiplying Eq. (13b) by $\mathbf{z}_{0}$, one can obtain

$v_{\alpha z}=\frac{q_{\alpha}}{m_{\alpha}} \frac{k_{z} \psi}{\omega-k_{y} v_{\alpha y}^{(0)}}$. 
Perturbation of the transverse velocity $\mathbf{v}_{\alpha \perp}$ is described by the equation

$$
\mathbf{v}_{\alpha \perp}=\frac{1}{\omega_{B \alpha}^{2}-\omega_{\alpha}^{\prime 2}} \frac{q_{\alpha}}{m_{\alpha}} \psi\left[\frac{i}{c} \frac{q_{\alpha}}{m_{\alpha}} \mathbf{k}_{\perp} \times \mathbf{B}-k_{\perp}\left(\omega-k_{y} v_{\alpha y}^{(0)}\right)\right] .
$$

Here we used the inequality $\left|v_{\alpha y}^{(0)} \kappa\right| \ll\left|\omega_{B \alpha}\right|$, where $\kappa$ is the inverse characteristic scale of the drift velocity (Eq. 14) variation along the axis $X, \omega_{B \alpha}=q_{\alpha} B /\left(m_{\alpha} c\right), \omega_{\alpha}^{\prime}=\omega-k_{y} v_{\alpha y}^{(0)}$.

Using the expressions (Eqs. 15, 17 and 18) one can calculate the disturbance of the $\alpha$-component concentration:

$$
n_{\alpha}=\psi n_{\alpha}^{(0)} \frac{q_{\alpha}}{m_{\alpha}}\left(\frac{k_{z}^{2}}{\omega_{\alpha}^{\prime 2}}-\frac{k_{y}^{2}}{\omega_{B \alpha}^{2}-\omega_{\alpha}^{\prime 2}}\right)-\frac{k_{y}}{\omega_{\alpha}^{\prime}} \frac{q_{\alpha} c}{m_{\alpha}} \psi \frac{\partial}{\partial x}\left[\frac{n_{\alpha}^{(0)} \omega_{B \alpha}}{\omega_{B \alpha}^{2}-\omega_{\alpha}^{\prime 2}}\right] .
$$

Assuming $\left|\omega_{\alpha}^{\prime}\right| \ll\left|\omega_{B \alpha}\right|$ and substituting expressions for $n_{\mathrm{e}}$ and $n_{\mathrm{i}}$ to the Poisson equation (13c), we obtain the following general dispersion relation:

$$
\begin{gathered}
k_{y}^{2}\left(1+\frac{\omega_{\mathrm{pi}}^{2}}{\omega_{B \mathrm{i}}^{2}}+\frac{\omega_{\mathrm{pe}}^{2}}{\omega_{B \mathrm{e}}^{2}}\right)+k_{z}^{2}\left(1-\frac{\omega_{\mathrm{pi}}^{2}}{\omega_{\mathrm{i}}^{\prime 2}}-\frac{\omega_{\mathrm{pe}}^{2}}{\omega_{\mathrm{e}}^{\prime 2}}\right)- \\
\frac{k_{y}^{2}}{\omega_{B \mathrm{i}}} \frac{\partial}{\partial x}\left(\frac{\omega_{\mathrm{pi}}^{2}}{\omega_{B \mathrm{i}}}\right) \frac{g_{\mathrm{e} x} m_{\mathrm{e}} / m_{\mathrm{i}}+g_{\mathrm{i} x}}{\omega_{\mathrm{i}}^{\prime} \omega_{\mathrm{e}}^{\prime}}=0 .
\end{gathered}
$$

Below this basic relation will be applied to the MHD stability analysis of the background plasma in the Jovian magnetosphere.

\subsection{Model dispersion relation in the case of bounded inho- mogeneous plasma}

It is clear that the dispersion relation (Eq. 20) is suitable for a plasma, which is uniform along the $Z$ axis. However, plasma distribution along the magnetic field line in the Jovian magnetosphere is not uniform: there are extensive regions of relatively rarefied plasma between the ionosphere and the dense disk in the vicinity of the magnetic equator.

Another very important circumstance is that any magnetic tube will lean on the conducting ionosphere. This, generally, can significantly damp a flute instability.

To take into account the above features, the following model of the background plasma disk in the Jovian magnetosphere is considered. We assume that the magnetic field lines are straight and bounded by the ionosphere with the given properties. In the middle of this plane cavity of the thickness $2 l$ a dense plasma disk of the given thickness $2 d$ (medium I) is placed, the rest of the cavity is filled with relatively rarefied plasma (medium II, see Fig. 5). It is assumed that the steady-state plasma density is homogeneous along the $Z$ axis both in media I and II, and their ratio does not depend on the distance from Jupiter: $n_{\mathrm{II}}^{(0)}=\tau n_{\mathrm{I}}^{(0)}$, where $\tau \leq 1$. This simplified model corresponds qualitatively to the magnetospheric conditions.

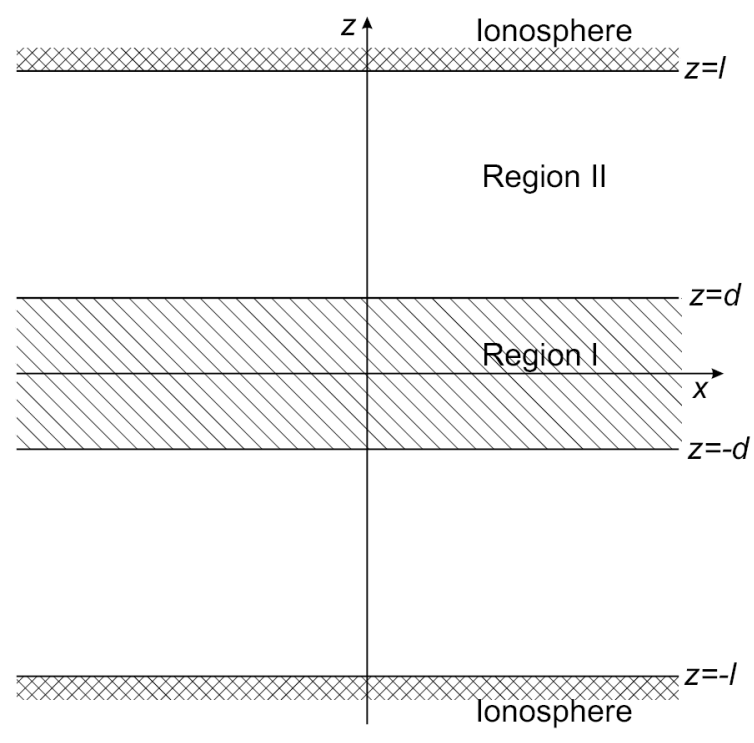

Fig. 5. A model field-aligned distribution of the background plasma. The magnetic field lines are straight and directed along the $z$ axis, the hatched region corresponds to the dense plasma disk (region I), there are regions of rarefied plasma between the disk and ionosphere (regions II). The external forces act along the $x$ axis, and in the case of the small-scale perturbations, Cartesian coordinates can be used, where the $y$ axis corresponds to the azimuth direction.

Since the projection of the external force onto the magnetic field line is maximum near the top of the line, for simplicity sake we neglect the force in the medium II. A total force acting upon the plasma components is caused, first of all, by the azimuthal rotation of the whole disk with the velocity $V \approx \Omega_{\mathrm{J}} x$ for $x \leq 15 R_{J}$ and $V \approx 15 R_{\mathrm{J}} \Omega_{\mathrm{J}}$ for $x>15 R_{\mathrm{J}}$

(Belcher, 1983), where $\Omega_{\mathrm{J}}=1.76 \cdot 10^{-4} \mathrm{rad} / \mathrm{s}$ is an angular velocity of the planetary rotation. Centrifugal acceleration in this case is the same both for ions and electrons, and for $x \geq 15 R_{\mathrm{J}}$ is equal to $g_{x}^{\mathrm{cf}} \sim \frac{\left(15 R_{\mathrm{J}} \Omega_{\mathrm{J}}\right)^{2}}{x} \approx 5 \cdot 10^{2} \frac{R_{\mathrm{J}}}{x} \mathrm{~m} / \mathrm{s}^{2}$. As follows from the last term in the left-hand side of Eq. (20), in this case an ion term dominates and should only be taken into account.

In accordance with the Jovian paraboloid magnetospheric magnetic field model there is a significant curvature of the magnetic field lines inside the disk. The radius of the curvature is about $2 R_{\mathrm{J}}$ and slightly depends on the radial distance $x$. Usually the effect of the curvature of the magnetic field lines is taken into account by an additional centrifugal acceleration $g_{\alpha x}^{\text {cur }}=\frac{v_{T_{\alpha}}^{2}}{R_{\mathrm{J}}} \sim \frac{T_{\alpha}}{m_{\alpha} R_{\mathrm{J}}}$, where $T_{\alpha}$ is the kinetic temperature of the $\alpha$-component. As seen, assuming electron and ion temperatures are equal: $T_{\mathrm{e}} \approx T_{\mathrm{i}}=T \approx 100 \mathrm{eV}$, both electron and ion contributions should be taken into account: $g_{\mathrm{e} x}^{\text {cur }} m_{\mathrm{e}} / m_{\mathrm{i}}+g_{\mathrm{i} x}^{\text {cur }} \sim \frac{T}{m_{i} R_{\mathrm{J}}} \approx 8 \mathrm{~m} / \mathrm{s}^{2}$. As seen, the effect of the magnetic field line curvature can be neglected as compared to the influence of the cold plasma disk rotation. 
Now let us estimate the parameters of the dispersion relation (Eq. 20) for medium I and II using measured values for the distance $15 R_{\mathrm{J}}: n_{\mathrm{I}}^{(0)} \sim 10^{-5} \mathrm{~m}^{-3}, B \sim 50 \mathrm{nT}$. The following inequality is met: $\omega_{\text {piI }}^{2} / \omega_{B \mathrm{i}}^{2} \sim 10^{7} \gg 1$. The corresponding relation is also valid in the medium II under any reasonable $\tau$. Taking into account that $1 \ll \omega_{\mathrm{piI}}^{2} / \omega_{\mathrm{i}}^{\prime 2} \ll \omega_{\mathrm{peI}}^{2} / \omega_{\mathrm{e}}^{\prime 2}$, one can obtain the dispersion relation in the medium I:

$k_{y}^{2} \frac{\omega_{\mathrm{piI}}^{2}}{\omega_{B \mathrm{i}}^{2}}-k_{z}^{2} \frac{\omega_{\mathrm{peI}}^{2}}{\omega_{\mathrm{e}}^{\prime 2}}-\frac{k_{y}^{2}}{\omega_{B \mathrm{i}}} \frac{\partial}{\partial x}\left(\frac{\omega_{p \mathrm{iI}}^{2}}{\omega_{B \mathrm{i}}}\right) \frac{\left(15 \Omega_{\mathrm{J}} R_{\mathrm{J}}\right)^{2}}{x \omega_{\mathrm{i}}^{\prime} \omega_{\mathrm{e}}^{\prime}}=0$.

Using the above assumption that the term with $\mathbf{g}_{\alpha}$ is equal to zero in the medium II, one can also write out a dispersion relation of the flute disturbances outside the disk:

$\omega^{2}=\frac{k_{z}^{2}}{k_{y}^{2}} \omega_{B \mathrm{i}}^{2} \frac{m_{\mathrm{i}}}{m_{\mathrm{e}}}$.

One can see that the system (Eqs. 21, 22) is not complete: it is necessary to determine the possible longitudinal wave number $k_{z}$. Let us construct an appropriate characteristic relation.

Disturbed electric potential $\psi_{1}$ in the medium I and $\psi_{2}$ in the medium II we present as two waves propagating in the opposite directions (generally, frequencies and wave vectors of the disturbances in both media can be different). Here we restrict ourselves by even modes, since their electric field is zero in the vicinity of the disk center, and one can expect that the lowest even modes have maximum increments since the damping influence of the conducting ionosphere is at aminimum.

To obtain a characteristic relation, the following boundary conditions at $z=d$ and $z=l$ were used. First, the transverse electric field at the boundaries should be continuous:

$\mathbf{E}_{\tau 1}=\mathbf{E}_{\tau 2}$.

Second, since the continuity equation $\operatorname{div}(4 \pi \mathbf{j}+i \omega \mathbf{E})=0$ is met, the normal component of the vector $4 \pi \mathbf{j}+i \omega \mathbf{E}$ should be continuous:

$4 \pi j_{z}+i \omega E_{z}=$ const.

Here, for simplicity sake, we also assume that the ionosphere is a perfect conductor ${ }^{1}$. Using the relations (Eqs. 23, 24), and the dispersion relation (Eq. 22) in the medium II, we obtain the following characteristic relation for the longitudinal wave number $k_{1 z}$ in the disk:

$k_{1 z} \tan \left(k_{1 z} d\right)=\frac{n_{\mathrm{II}}^{(0)}}{n_{\mathrm{I}}^{(0)}} \frac{\omega k_{y}}{\sqrt{\omega_{B \mathrm{i}}\left|\omega_{B \mathrm{e}}\right|}} \cot \left(\frac{\omega k_{y}(l-d)}{\sqrt{\omega_{B \mathrm{i}}\left|\omega_{B \mathrm{e}}\right|}}\right)$.

The above expression (Eq. 25) and dispersion relation for the medium I (Eq. 21), where $k_{z}=k_{z 1}$, describe a flute perturbation in the plasma disk.

\footnotetext{
${ }^{1}$ More detailed survey shows that this assumption is valid under the condition $\left|k_{y}\right| \gg \frac{1}{4 \pi \Sigma_{\mathrm{P}}} \frac{\omega_{\text {peII }}^{2}}{\omega_{B \mathrm{i}} \sqrt{m_{\mathrm{i}} / m_{\mathrm{e}}}}$, where $\Sigma_{\mathrm{P}}$ is the heightintegrated Pedersen conductivity of the Jovian ionosphere.
}

4.3 Threshold radial profile of the background plasma density

It was discussed above that we need to investigate threshold conditions of the flute instability in the plasma disk. It can be assumed that the most unstable modes correspond to the smallest longitudinal wave numbers. So, we shall search for a solution which satisfies the condition

$k_{1 z} d \ll 1$.

An analysis of the flute instability under the condition $k_{1 z} d=0$ (see Bespalov and Davydenko, 1994) shows that unstable modes have very low real frequencies, so we can also suppose that an argument of the trigonometric function in the right-hand side of Eq. (25) is also small enough:

$\frac{\omega\left|k_{y}\right|(l-d)}{\sqrt{\omega_{B \mathrm{i}}\left|\omega_{B \mathrm{e}}\right|}} \ll 1$.

Using the conditions $(26,27)$, the functions in Eq. (25) can be expanded, and the small terms can be neglected. Substituting this equation in formula (21) and neglecting the drift of the electrons as compared to ion's drift $\left(\omega_{\mathrm{e}}^{\prime} \approx \omega\right)$, we obtain the following reduced characteristic relation:

$\omega^{2} \omega_{\mathrm{i}}^{\prime}-\frac{V^{2} \omega}{x} \frac{\partial}{\partial x}\left(\ln \frac{n_{\mathrm{I}}^{(0)}}{B}\right)-\frac{\omega_{B \mathrm{i}}^{2}}{k_{y}^{2}} \frac{m_{\mathrm{i}}}{m_{\mathrm{e}}} \frac{\omega_{\mathrm{i}}^{\prime} \tau}{d(l-d)}=0$.

It is seen that the relation (Eq. 28) presents a cubic equation with respect to the frequency $\omega$ :

$\omega^{3}+\mathcal{A} \omega^{2}+\mathcal{B} \omega+\mathcal{C}=0$,

where

$\mathcal{A}=\frac{V^{2}}{\omega_{B i}} \frac{k_{y}}{x}$,

$\mathcal{B}=-\frac{V^{2}}{x} \frac{\partial}{\partial x}\left(\ln \frac{n_{\mathrm{I}}^{(0)}}{B}\right)-\frac{\omega_{B \mathrm{i}}^{2}}{k_{y}^{2}} \frac{m_{\mathrm{i}}}{m_{\mathrm{e}}} \frac{\tau}{2 R_{\mathrm{J}}\left(1.3 x-2 R_{\mathrm{J}}\right)}$,

$\mathcal{C}=-\frac{\omega_{B \mathrm{i}}}{k_{y}} \frac{m_{\mathrm{i}}}{m_{\mathrm{e}}} \frac{\tau}{2 R_{\mathrm{J}}\left(1.3 x-2 R_{\mathrm{J}}\right)} \frac{V^{2}}{x}$,

where the thickness of the plasma disk is about $4 R_{\mathrm{J}}, l \approx 1.3 x$.

Starting conditions of the flute instability correspond to the appearance of the complex roots of Eq. (29), i.e. on the threshold of the instability the determinant of Eq. (29) should be zero:

$\left[-\mathcal{A}^{2} / 3+\mathcal{B}\right]^{3} / 27+\left[2(\mathcal{A} / 3)^{3}-\mathcal{A B} / 3+\mathcal{C}\right]^{2} / 4=0$.

One can see that at a given radial distance $x$ the condition (Eq. 30) itself presents the cubic equation with respect to the relative steepness of the radial profile of the plasma density $\partial\left(n_{\mathrm{I}}^{(0)} / B\right) / \partial x$. The only single parameter of the perturbations in Eq. (30) is the transverse wave number $k_{y}$. 


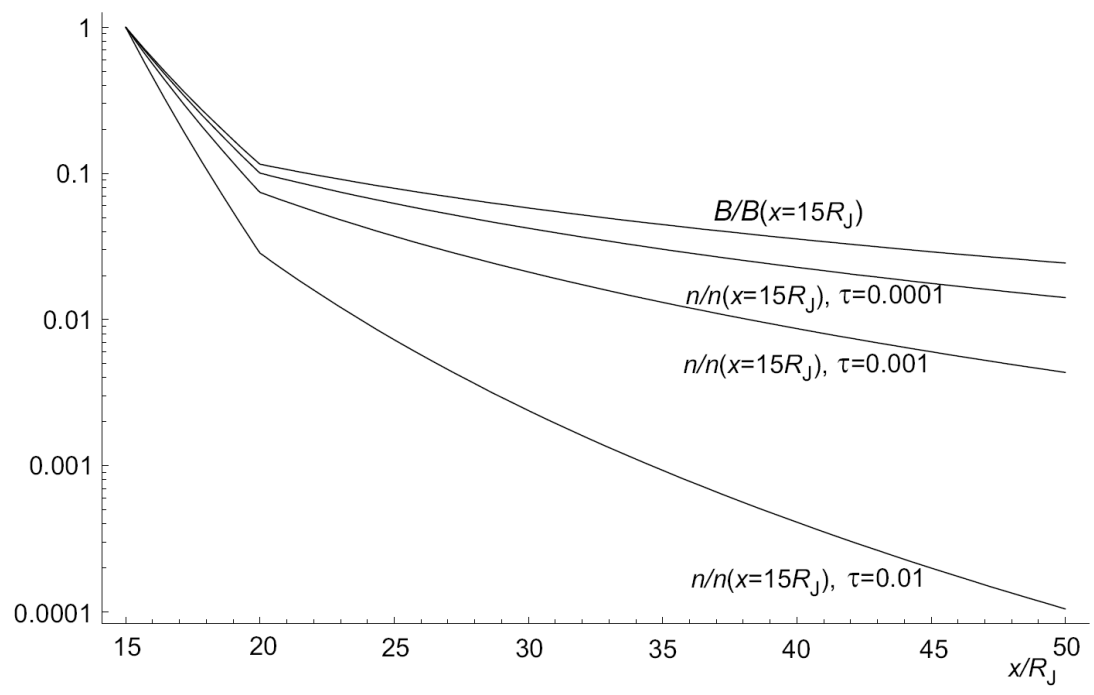

Fig. 6. Normalized model radial distributions of the magnetic field strength in the plasma disk (see Sect. 2) and background plasma density under $\tau=0.01,0.001$, and 0.0001 . Here $B\left(x=15 R_{\mathrm{J}}\right)$ and $n\left(x=15 R_{\mathrm{J}}\right)$ are the magnetic field strength and plasma density at the radial distance $x=15 R_{\mathrm{J}}$, respectively.

An numerical analysis shows that the determinant of Eq. (30) is always positive, i.e. this equation has only one real solution for $\partial\left(n_{\mathrm{I}}^{(0)} / B\right) / \partial x$ at a given $x$ and $k_{y}$. This root is always negative, since at the threshold of the flute instability a plasma density decreases with $x$ faster than the magnetic field, due to the damping effect of the conducting ionosphere (it is known that the threshold relative steepness in the case of the boundless plasma is equal to zero, i.e. the threshold's radial profile of the plasma density repeats the radial distribution of the magnetic field). It is natural to assume that the most unstable mode corresponds to the maximum relative steepness: there are bands of unstable modes under the steeper density profile and there are no unstable flute perturbations under the smoother density profile. This value determines the plasma density profile, which corresponds to the appearance of the first unstable mode and depends on the parameter $\tau$ and the magnetic field distribution only. ${ }^{2}$ Substituting the value of the threshold relative steepness $\partial\left(n_{\mathrm{I}}^{(0)} / B\right) / \partial x$ to Eq. (30) one can determine the transverse wave number of the most unstable mode. Using Eq. (29) we can also determine the frequency of this mode.

The radial distribution of the magnetic field in the equatorial disk agrees with the above paraboloid Jovian magnetospheric magnetic field model and is presented in Fig. 6. The calculated threshold radial profiles of the plasma density for different density ratio $\tau$ are also presented in Fig. 6. In accordance with qualitative expectations, an influence of the

\footnotetext{
2 The transverse wave number and the frequency of the most unstable mode should satisfy the above conditions $\left|\omega_{\alpha}^{\prime}\right| \ll\left|\omega_{B \alpha}\right|$ and (27). It should be noticed that the last condition is satisfied under $\tau \lesssim 0.1$ only, and the case $\tau \geq 0.1$ needs more specific analysis and was not considered.
}

conducting ionosphere becomes less (and the density profile tends to the radial distribution of the magnetic field in the disk) as $\tau$ decreases, since the plasma outside the disk becomes more tenuous and conditions at the disk boundary become close to free (vacuum) conditions. In accordance with the calculations, under the density ratio $\tau=10^{-4}$, the threshold plasma density profile almost exactly corresponds to the magnetic field distribution which is typical for the case of plasma disk in a free space. If the ratio of the plasma densities outside and inside the disk is $\tau=10^{-3}$, then the threshold plasma density profile can be described by the expression $n_{\mathrm{I}}^{(0)}(x)=\Phi(x, \tau) B(x) x^{-1.4}$, where $\Phi(x, \tau) \sim 1$. If $\tau=10^{-2}$, the model profile corresponds to $n_{\mathrm{I}}^{(0)}(x)=\Phi(x, \tau) B(x) x^{-4.4}$.

In accordance with the in-situ measurements in the Jovian plasma disk, the plasma density and magnetic field distribution are both approximately described by the power laws (Belcher, 1983; Khurana and Kivelson, 1993). The plasma density profile is steeper, and the difference in the magnetic field and plasma density exponents lies in the range between 1 and 2 . One can see that the above results qualitatively agree with the experimental data. The density ratio $\tau$ plays the role of a free parameter, which can be varied to met the calculated and observed radial profiles of the background plasma density in the disk. Thus, the value $\tau$ can also provide additional information on the plasma density outside the disk: the best coincidence of the experimental and calculated profiles reaches the density ratio $\tau \approx 10^{-3}$. This result qualitatively agrees with the direct plasma measurements (see Belcher, 1983).

It should be noticed that the above approach can be applied to the analysis of the radial plasma density profiles in the stable regions of other planetary magnetospheres. In particular, 
this method can be applied to the rather stable inner part of Saturn's magnetosphere. The stability of the background plasma in the outer, unstable part of Saturn's magnetosphere needs specific consideration (see Goertz, 1983).

\section{Conclusions}

The paraboloid model of Jupiter's magnetospheric magnetic field was used for calculations of the open field line region dependent on the IMF, and for determination of the radial distribution of the background plasma in the Jovian magnetodisk.

The predictions made on the basis of the Jovian paraboloid model were compared with observations. We showed that the main oval radius is of the order of $16^{\circ}$, which corresponds to observations. The equatorward boundary of the main oval in our model is an ionospheric projection of the inner edge of magnetodisk, where the maximum field-aligned electric potential drops along upward field-aligned currents, which occur due to the maximum magnetic field lines slippage.

The regions of different corotation regimes in the highlatitude Jovian ionosphere were determined to be dependent on the IMF orientation. For southward IMF, at ionospheric latitudes less than $\sim \pm 74^{\circ}$, the rigid corotation exists; from $\sim \pm 74^{\circ}$ to $\sim \pm 84^{\circ}$ the sub-corotation should take place; at latitudes higher than $\sim \pm 84^{\circ}$ the ionosphere does not rotate. For northward IMF, at ionospheric latitudes less than $\sim \pm 74^{\circ}$ the rigid corotation exists; from $\sim \pm 74^{\circ}$ to $\sim \pm 81^{\circ}$ the subcorotation should take place; for higher latitudes the rotation should be restored.

Following Grodent et al. (2003b), we suggested that the swirl region is associated with an open Jovian field line region at the ionospheric level. It was shown by observations that the swirl region occupies approximately one-third the area limited by the main auroral oval, and that motions inside it occasionally form localized swirls, though the sense of rotation is not clear and varies from one data set to the other. The area of the open field line region in the highlatitude ionosphere calculated in the paraboloid model for the typical IMF of $0.5 \mathrm{nT}$ was also of the order of one third from the area of a region bounded by the ionospheric projection of the inner edge of the magnetodisk (which we consider to be coincident with the equatorward boundary of the main oval). According to our calculations, different vortex structures are formed inside the ionospheric open field line region, dependent on the IMF orientation. For southward IMF, ionospheric convection inside the open field line region forms two cells for the IMF component $B_{I M F y}=0$ (see Fig. 4), and one cell significantly dominates for $B_{I M F y} \neq 0$ (for $B_{I M F y} \neq 0$ and $B_{I M F x} \neq 0$ there are two unequal cells; for $B_{I M F x}=0$ there is only one vortex). Dependent on the sign of $B_{I M F y}$ we can see, for example, on the dawn side of the northern ionospheric open field line region, the clockwise or anticlockwise motions. For northward IMF, according to our scenario, there is a corotation inside the open field line region.

The elaborated model of the magnetic field was used to determine the radial profile of the background plasma density in the magnetospheric disk. The dense plasma of the rotating disk is a subject of flute instability, which can provide fast transfer of the iogenic plasma to the periphery of the magnetosphere. Considering the instability of the disk plasma, a coupling between the dense disk, the tenuous plasma outside the disk, and the conducting planetary ionosphere was taken into account. Assuming that the plasma in the disk is at the instability threshold to the flute perturbations, the limiting radial plasma density profiles were calculated. In accordance with the previous results, when the density ratio at the disk boundary is small enough $\left(\tau \sim 10^{-5}\right)$, the radial profiles of the plasma density and the magnetic field almost coincide. As $\tau$ grows, the density profile appears to be steeper relative to the magnetic field's profile (due to the mitigating influence of the conducting ionosphere), and approximately fits the experimental results at $\tau \sim 10^{-3}$.

Acknowledgements. This work was partly supported by the INTAS Grant No. 03-51-3922 and by the RFBR Grants No 04-05-64396, No 05-05-64435 and No 05-02-16350. Authors thank the referees for providing useful commentary. We also thank I. I. Alexeev for help in processing the simulation results.

Topical Editor T. Pulkkinen thanks R. Prangé and T. Karlsson for their help in evaluating this paper.

\section{References}

Alexeev, I. I.: The penetration of interplanetary magnetic and electric fields into the magnetosphere, J. Geomag. Geoelectr., 38, 1199-1221, 1986.

Alexeev, I. I., and Belenkaya E. S.: Modeling of the Jovian Magnitosphere, Ann. Geophys., 23, 809-826, 2005.

Ballester, G. E., Clarke, J. T., Trauger, J. T., et al.: Time-resolved observations of Jupiter's far-ultraviolet aurora, Science, 274, 409-412, 1996.

Barbosa, D. D., Gurnett, D. A., Kurth, W. S., and Scarf, F. L.: Structure and properties of Jupiter's magnetoplasmadisc, Geophys. Res. Lett., 6, No 10, 785-788, 1979.

Barish, F. D. and Smith, R. A.: An analytical model of the jovian magnetosphere shape, Geophys. Res. Lett., 2, 269-272, 1975.

Beard, D. B. and Jackson, D. J.: The jovian magnetic field and magnetosphere, J. Geophys. Res., 81, 3399-3401, 1976.

Belcher, J. W.: The low-energy plasma in the Jovian magnetosphere, in: Phycics of the Jovian Magnetosphere, Cambridge, Plan. Sci. ser, edited by: Dessler, A. J., Cambrige University Press, New York, 68-105, 1983.

Belenkaya, E. S.: Peculiarities of the interaction between the solar wind magnetic field with the terrestrial and jovian magnetospheres, Geomagn. and Aeronom (in Russian), 43, No 2, 174 182, 2003.

Belenkaya, E. S.: The jovian magnetospheric magnetic and electric fields: effects of the interplanetary magnetic field, Planet. Space Sci., 52, 499-511, 2004. 
Bespalov, P. A. and Zheleznyakov, V. V.: Formation of disks around hot magnetic stars under the action of radiation pressure, Sov. Astron. Lett., 16, 442-449, 1990.

Bespalov, P. A. and Davydenko, S. S.: On the structure of the plasma disk in the Jovian magnetosphere, Planet. Space Sci., 42, No.7, 583-592, 1994.

Bridge, H. S., Belcher, J. W., Lazarus, A. J., Sillivan, J. D., McNutt, R. L. Jr., Bagenal, F., Scudder, J. D., Sittler, E. C., Siscoe, G. L., Vasyliunas, V. M., Goertz, C. K., and Yeates, C. M.: Plasma observations near Jupiter: Initial results from Voyager 1. Science, 204, 987-991, 1979a.

Bridge, H. S., Belcher, J. W., Lazarus, A. J., Sillivan, J. D., Bagenal, F., McNutt Jr., R. L., Ogilvie, K., Scudder, J. D., Sittler, E. C., Vasyliunas, V. M. and Goertz, C. K.: Plasma observations near Jupiter: Initial results from Voyager 2, Science, 206, 972-976, 1979b.

Bunce, E. J. and Cowley S. W. H.: Divergence of the equatorial current in the dawn sector of Jupiter's magnetosphere, Planet. Space Sci., 49, 1089-1113, 2001.

Caudal, G.: A self-consistent model of Jupiter's magnetodisc including the effects of centrifugal force and pressure, J. Geophys. Res., 91, 4201-4221, 1986.

Clarke, J. T., Ballester, G. E., Trauger, J., Evans, R., Connerney, J. E. P., Stapelfeldt, K., Crisp, D., Feldman, P. D., Burrows, C. J., Casertano, S., Gallagher III, J. S., Griffiths, R. E., Hester, J. J., Hoessel, J. G., Holtzman, J. A., Krist, J. A., Meadows, V., Mould, J. R., Scowen, P. A., Watson, A. M., and Westphal, J. A.: Far-ultraviolet imaging of Jupiter's aurora and the Io "footprint", Science, 274, 404-409, 1996.

Clarke, J. T., Ballester, G., Trauger, J., Ajello, J., Pryor, W., Tobiska, K., Connerney, J. E. P., Gladstone, G. R., Waite Jr., J. W., Jaffel, L. B., and Gerard, J.-C.: Far-UV imaging of Jupiter's aurora with HST/WFPC 2, Hubble Space Telescope imaging of Jupiter's UV aurora during the Galileo orbiter mission, J. Geophys. Res., 103, E9, 20 217-20 236, 1998.

Connerney, J. E. P.: Doing more with Jupiter's magnetic field, in: Planetary Radio Emission III, edited by: Rucker, H. O., Bauer, S. J., and Kaiser, M. K., Austrian Acad. Sci., Vienna, 13-33, 1992.

Connerney, J. E. P.: Magnetic field of the outer planets, J. Geophys. Res., 98, 18 659-18 679, 1993.

Connerney, J. E. P., Acuña, M. H., Ness, N. F., and Satoh, T.: New models of Jupiter's magnetic feld constrained by the Io flux tube footprint, J. Geophys. Res., 103, N A6, 11 929-11 940, 1998.

Cowley, S. W. H. and Bunce, E. J.: Origin of the main auroral oval in Jupiter's coupled magnetosphere-ionosphere system, Planet. Space Sci., 49, 1067-1088, 2001.

Cowley, S. W. H., Bunce, E. J., Stallard, T. S., and Miller, S.: Jupiter's polar ionospheric flows: Theoretical interpretation, Geophys. Res. Lett., 30(5), 1220, doi:10.1029/2002GL016030, 2003.

Engle, I. M.: Idealized Voyager Jovian magnetosphere shape and field, J. Geophys. Res., 96, No A5, 7793-7802, 1991.

Engle, I. M.: The field of the Jovian magnetosphere, including contributions of the magnetopause surface currents, Advances in Space Research, 12, No 8, 249-255, 1992a.

Engle, I. M.: Diurnal variation in Jovian subsolar magnetopause position, J. Geophys. Res., 97, No A11, 17 169-17 172, 1992b.

Engle, I. M. and Beard, D. B.: Idealized Jovian magnetosphere shape and field, J. Geophys. Res., 85, 579-592, 1980.
Ferriere, K. M. and Andre, N.: A mixed magnetohydrodynamickinetic theory of low-frequency waves and instabilities in stratified, gyrotropic, two-component plasmas, J. Geophys. Res., 108, No A7, 1308, doi:10.1029/2003JA009883, 2003.

Fukazawa, K., Ogino, T., and Walker, R. J.: Dynamics of the Jovian magnetosphere for northward interplanetary magnetic field (IMF), Geophys. Res. Lett., 32, L03202, doi:10.1029/2004GL021392, 2005.

Goertz, C. K.: Io's interaction with the plasma torus, J. Geophys. Res., 85, 2949-2956, 1980.

Goertz, C. K.: Detached plasma in Saturn's front side magnetosphere, Geophys. Res. Lett., 10, 6, 455-458, 1983.

Goldstein, M. L., Lepping, R. P., and Sittler Jr., A. C.: Magnetic field properties of Jupiter's tail at distances from 80 to 7500 Jovian radii, J. Geophys. Res., 90, 8223-8239, 1985.

Goldstein, M. L., Lepping, R. P., and Sittler Jr., A. C.: Reply, J. Geophys. Res., 91, 7133, 1986.

Grodent, D., Clarke, J. T., Kim, J., Waite, J. H., and Cowley, S. W. H.: Jupiter's main auroral oval observed with HST-SNIS, J. Geophysical Res., 108, No A11, 1389, doi:10.1029/2003JA009921, 2003a.

Grodent, D., Clarke, J. T., Waite, J. H., Cowley, S. W. H., Gerard, J.C., and Kim, J.: Jupiter's polar auroral emission, J. Geophysical Res., 108, No A10, 1366, doi:10.1029/2003JA010017, 2003b.

Hawkins III, S. E., Cheng, A. F., and Lanzerotti, L. J.: Bulk flows of hot plasma in the Jovian magnetosphere: A model of anisotropic fluxes of energetic ions, J. Geophys. Res., 103, 20 031-20 054, 1998.

Hill, T. W.: Interchange stability of rapidly rotating magnetosphere, Planet. Space Sci., 24, 1151-1154, 1976.

Hill, T. W.: The Jovian auroral oval, J. Geophys. Res., 106, 81018107, 2001.

Kane, M., Mauk, B. H., Keath, E. P., and Krimigis, S. M.: Hot ions in Jupiter's magnetodisk: A model for Voyager 2 low energy charged particle measurements, J. Geophys. Res., 100, 1947319486, 1995.

Kane, M., Williams, D. J., Mauk, B. H., McEntire, R. W., and Roelof, E. C.: Galileo energetic particles detector measurements of hot ions in the neutral sheet region of Jupiter's magnetodisk, Geophys. Res. Lett., 26, 5-8, 1999.

Khurana, K. K.: Euler potential models of Jupiter's magnetospheric field, J. Geophys. Res., 102, 11 295-11 306, 1997.

Khurana, K. K. and Kivelson, M. G.: Inference of the angular velocity of plasma in the jovian magnetosphere from the sweepback of magnetic field, J. Geophys. Res., 98, 67-80, 1993.

Kivelson, M. G. and Southwood, D. J.: First evidence of IMF control of Jovian magnetospheric boundary locations: Cassini and Galileo magnetic field measurements compared, Planet. Space Sci., 51, 891-898, 2003.

Krimigis, S. M., Armstrong, T. P., Axford, W. I., Bostrom, C. O., Fan, C. Y., Gloeckler, G., Lanzerotti, L. J., Keath, E. P., Zwickl, R. D., Carbary, J. F., and Hamilton, D. C.: Low-energy charged particle environment at Jupiter - a first look, Science, 204, 9981002, 1979a.

Krimigis, S. M., Armstrong, T. P., Axford, W. I., Bostrom, C. O., Fan, C. Y., Gloeckler, G., Lanzerotti, L. J., Keath, E. P., Zwickl, R. D., Carbary, J. F., and Hamilton, D. C.: Hot plasma environment at Jupiter: Voyager 2 results, Science, 206, 977-984, $1979 b$. 
Laxton, N. F., Balogh, A., Cowley, S. W. H., Dunlop, M. W., Forsyth, R. J., Hynds, R. J., and Staines, K.: Origins of the first-order anisotropy of $1 \mathrm{MeV}$ protons in the Jovian magnetosphere during the Ulysses flyby: Flux gradients and plasma flows, Planet. Space Sci., 45, 1143-1170, 1997.

Liu, W. W.: Centrifugally Driven Instability of a Rotationally Dominated Magnetodisk, J. Geophys. Res., 103, No. A3, 4707-4714, 1998.

McKibben, R. B. and Simpson, M. A.: Evidence from charged particle studies for the distortion of the Jovian magnetosphere. J. Geophys. Res., 79, 3545-3554, 1974.

Melrose, D. B.: Rotational effects on the distribution of thermal plasma in the magnetosphere of Jupiter, Planet. Space Sci., 15, 381-393, 1967.

Mikhailovskii, A. B.: Theory of Plasma Instabilities. Vol.1. Instabilities of an Inhomogeneous Plasma, Consultants Bureau, New York - London, 1974.

Pallier, L. and Prangé, R.: More about the structure of the high latitude Jovian aurorae, Planetary Space Sci., 49, 1159-1173, 2001.

Prangé, R., Rego, D., Pallier, L., Connerney, J. E. P., Zarka, P., and Queinnec, J.: Detailed study of FUV Jovian auroral features with the post-COSTAR HST faint object camera, J. Geophys. Res., 103, N E9, 20 195-20 215, 1998.

Prangé, R., Chagnon, G., Kivelson, M. G., Livengood, T. A., and Kurth, W.: Temporal monitoring of Jupiter's auroral activity with IUE during the Galileo mission. Implications for magnetospheric processes, Planetary Space Sci., 49, 405-415, 2001.
Rego, D., Achilleous, N., Stallard, T., Miller, S., Prangé, R., Dougherty, M., and Joseph, R. D.: Supersonic winds in Jupiter's aurora, Nature, 399, 121-124, 1999.

Siscoe, G. L. and Summers, D.: Centrifugally driven diffusion of Iogenic plasma. J. Geophys. Res., 86, No A10, 8471-8479, 1981.

Smith, E. J., Davis Jr., L., Jones, D. E., Colburn, D. S., Coleman Jr., P. J., Dyal, P., and Sonett, C. P.: Magnetic field of Jupiter and its interaction with Solar wind, Science, 183, 305-306, 1974.

Southwood, D. J. and Kivelson, M. G.: A new perspective concerning the influence of the solar wind on the jovian magnetosphere, J. Geophys. Res., 106, 6123-6130, 2001.

Stallard, T. S., Miller, S., Milward, G., and Joseph, R. D.: On the dynamics of the Jovian ionosphere and thermosphere, part 1, The measurement of ion winds, Icarus, 154, 475-491, 2001.

Stallard, T. S., Miller, S., Cowley, S. W. H., and Bunce, E. J.: Jupiter's polar ionospheric flows: Measured intensity and velocity variations poleward of the main auroral oval, Geophys. Res. Letts., 30, No 5, 1221, doi:10.1029/2002GL016031, 2003.

Tomas, A. T., Woch, J., Krupp, N., Lagg, A., Glassmeier, K.-H., and Kurth, W. S.: Energetic electrons in the inner part of the Jovian magnetosphere and their relation to auroral emissions, J. Geophys. Res., 109, A06203, doi:10.1029/2004JA010405, 2004.

Walker, R. J. and Ogino, T.: A simulation study of currents in the Jovian magnetosphere, Planet. Space Sci., 51, 295-307, 2003.

Walker, R. J., Kivelson, M. G., and Schardt, A. W.: High- $\beta$ plasma in the dynamic Jovian current sheet. Geophys. Res. Lett., 5, 799$802,1978$. 